\title{
Research On The Mapping Grinding of Dimple Surface With Ordered Pattern Based On Topological Theory
}

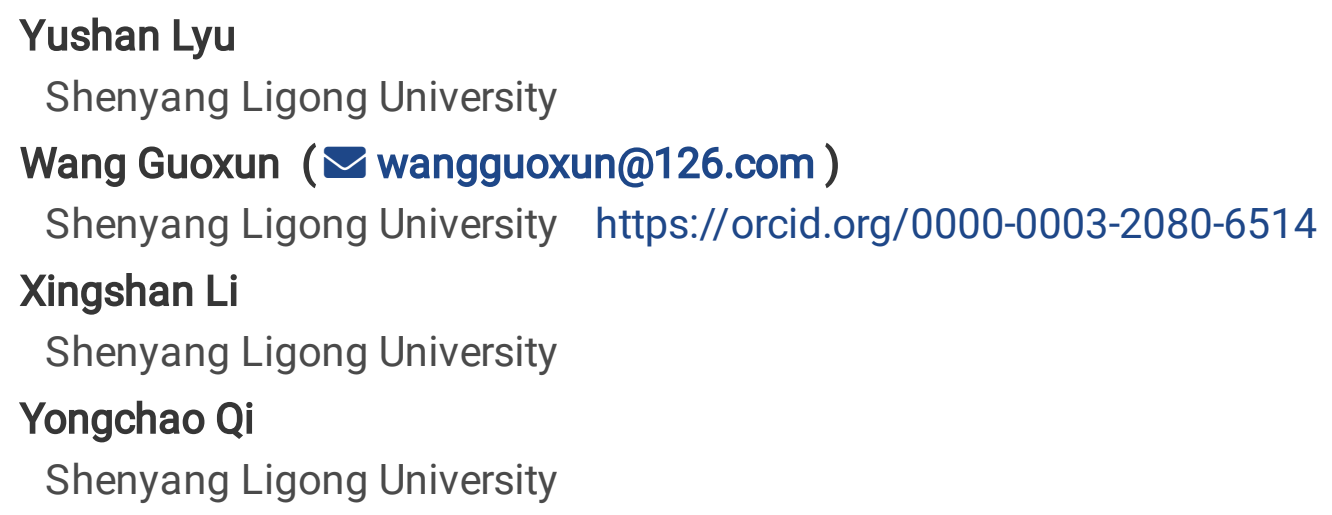

\section{Research Article}

Keywords: Grinding, Topological grinding, Structured dimple surface, Structured grinding wheel

Posted Date: January 27th, 2022

DOI: https://doi.org/10.21203/rs.3.rs-1281734/v1

License: (c) (i) This work is licensed under a Creative Commons Attribution 4.0 International License. Read Full License 


\title{
Research on the Mapping Grinding of Dimple Surface with Ordered Pattern \\ Based on Topological Theory
}

\author{
Yushan Lyu, Guoxun Wang*, Xingshan Li, Yongchao Qi \\ School of Mechanical Engineering, Shenyang Ligong University, Shenyang, 110159, China \\ *Corresponding author: Guoxun Wang, E-Mail: wangguoxun@126.com
}

\begin{abstract}
In order to grind structured dimple surface, a topological mapping grinding method for structured dimple surface was proposed based on grinding kinematics principle and point set topology. Firstly, the topological space of the workpiece and the grinding wheel was established, and the topological features of the structured dimple surface and the structured grinding wheel were extracted based on the analysis of the morphology features of the structured surface with array, staggered and phyllotactic pattern. Then, the topology mapping equation of the grinding process was constructed based on the analysis of the generating mechanism of the dimple surface about grinding geometry, and the structured grinding wheel was designed according to the topology mapping equation. Finally, according to the grinding geometry simulation, the influence of grinding parameters on the generating of dimple surface morphology was studied, and the grinding experiment was carried out. The results show that the structured grinding wheel designed according to the topological features of the structured dimple surface can achieve the grinding of the structured dimple surface. The ground dimple surface is a topological dimple surface, and the feature parameters of the dimple surface can be changed with the change of grinding parameters, but the topological feature properties remain unchanged under the condition of satisfying the proper grinding speed ratio.
\end{abstract}

Keywords: Grinding, Topological grinding, Structured dimple surface, Structured grinding wheel

\section{Introduction}

"Structured", "textured" or "engineered" surfaces are important for improving the performance of mechanical parts or products [1]. In recent years, with the continuous development of surface biomimetic technology, the theoretical research on structured surfaces has continued to deepen, and its surface morphology has also tended to be diverse [2, 3, 4]. In particular, the research on bionic structured drag reduction surfaces has attracted more and more attention from scholars and engineers, and has become one of the important research directions in the field of surface engineering design $[5,6,7,8]$. However, how to put the theoretical research results of structured drag reduction surfaces into practical engineering applications, the research on the high-efficiency or high-precision and low-cost manufacturing technology of the surface has become a bridge between theoretical research results and practical engineering. At present, the manufacturing methods of structured surfaces mainly include rolling method, imprinting method, electrolytic etching method, laser ablation method, photolithography mask method, cutting and grinding, etc. [9-14]. Among them, the grinding method is one of the economical or efficient manufacturing methods to realize the structured surface of difficult-to-machine material parts or the structured surface of mass-produced parts. In the field of structured surface grinding, its research is mainly reflected in the following aspects:

In the research of using spiral grooved grinding wheel to grind structured surface, Stepien Piotr 
[15-19] first proposed a method of dressing spiral grooves on the surface of the grinding wheel to grind the surface texture of grooves, convex-hulls and dimples. The corresponding structured surface was cut out, and the creation mechanism of the surface was studied in depth. On this basis, Kim H and Ko T J [20, 21, 22 ] used the CAM simulation method to design a parametric grinding wheel, and analyzed the influences of the grinding wheel dressing geometric parameters and grinding parameters on the surface morphology of the workpiece. Cao H Y [23] developed the dressing theory of spiral groove grinding wheel and the grinding control strategy of cylindrical structured surface. Liu YM [24] developed the influence law of the dressing spiral groove on the structured surface morphology and grinding force of the workpiece, and established the structured morphology and grinding force model of the workpiece. The model prediction results and the experimental results have good consistency. Mohamed AL-M [25] carried out the research on predicting the surface texture of the workpiece surface with the circumferential groove grinding wheel. Through the study of the influence of grinding parameters, grinding direction and groove coefficients on the structured surface height, spacing and angle on the surface structure, a surface prediction model was established to realize the prediction of structured surface morphology.

In order to realize the grinding of multi-form structured surfaces, Oliveira J F G. and Silva E J [26-29] proposed a dressing method for grinding wheel, and the grinding wheel dressed by this method was used to grind friction reduction surfaces with various structured morphologies such as triangle wave, groove, dimple and convex-hull, also provides the kinematic conditions for realizing structured surface grinding. Moreno M G [30] applied this research to the grinding of the friction reduction surface of the machine tool guide rail, and studied the friction performance of the ground surface to improve the wear resistance of the guide rail, which proved that the application of this grinding technology is feasible.

In the grinding of structured groove drag reduction surface, Denkena B [31-34] carried out the research of groove profile grinding based on the geometric morphology of the shark riblet surface. With the help of the shaping and dressing of the grinding wheel, the surface of the compressor blade realizes the grinding of the riblet surface, and the generating mechanism of the burrs during grinding process and the influence of the grinding wheel wear on the groove geometry are also deeply studied, and corresponding solutions are given. At the same time, a new grinding wheel with a nutria-like tooth structure was proposed. Xie $\mathrm{J}[35,36]$ carried out a research on the grinding of the micro-groove array on the rake face of titanium alloy cutting tools, which improved the cutting performance of the tool. Wu M T and Guo B [37] used lasers to structure the coarse-grained diamond grinding wheel and realized the micro-structure grinding on $\mathrm{SiC}$ material surface.

In terms of theoretical research on the design of grinding wheels for structured surface grinding, although in order to improve the grinding performance of the grinding wheel, the research on structured grinding wheels has made good progress [38], However, the theoretical research on the design of structured grinding wheels for grinding complex structured surfaces is relatively weak. Monie A and Guo B [39] conducted research on this problem and gave meaningful design strategies.

The above-mentioned research has promoted the development of structured surface grinding very well. However, it can also be seen that in addition to the profile grinding method that can design the grinding wheel according to the structured surface morphology, in most cases, the grinding wheel is dressed to a certain structured morphology, and then the structured surface morphology of the workpiece is obtained by selecting the grinding parameters appropriately, however, the research on designing a structured grinding wheel based on the morphological features of the structured surface and then grinding the corresponding structured surface is relatively weak, which make the study of the grinding theory of structured surface have a certain degree of passivity and is not conducive to the practical 
application of structured surface grinding. Therefore, based on the point set topology theory [40] and the principle of grinding kinematics, this paper proposes a topological mapping grinding strategy for structured dimple surface.

\section{Basic strategy of the topological grinding of dimple surface}

As shown in Fig. 1, suppose the coordinate system of the grinding wheel is $\boldsymbol{S}\left(O_{s}, X_{s}, Y_{s}, Z_{s}\right)$, the coordinate system of the workpiece is $W\left(O_{w}, X_{w}, Y_{w}, Z_{w}\right)$, and the center of $\boldsymbol{S}\left(O_{s}, X_{s}, Y_{s}, Z_{s}\right)$ is at the rotation center of the grinding wheel end face and the center of $W\left(O_{w}, X_{w}, Y_{w}, Z_{w}\right)$ is at the center of the first dimple being ground. In the grinding process of dimple surface, when the grinding parameters (grinding wheel speed $n_{s}$, feed speed $v_{w}$ and grinding depth $a_{p}$ ) meet the appropriate functional relationship, the abrasive cluster subset $S_{i j}$ on the grinding wheel surface with the base radius $R_{s}$ can remapped and ground out the dimple subset $W_{i j}$ on workpiece surface in a broad sense. In this process, the abrasives with randomly

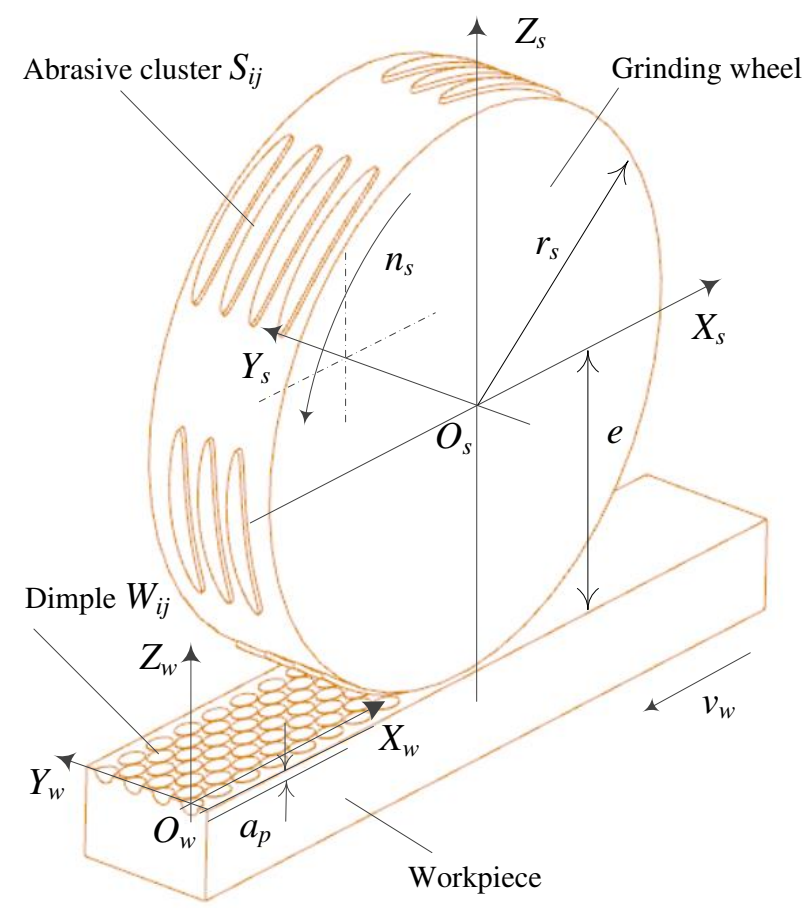

Fig. 1 Schematic diagram of the topological mapping of grinding the dimple surface with the structured wheel

distributed features in the abrasive cluster subset cut the workpiece surface with their respective cycloid trajectories, in which only the trajectory of the effective dynamic cutting edge can finally envelop the dimple subset $W_{i j}$. Therefore, in Euclidean space, it is very complicated and difficult in engineering to obtain the equation of the abrasive cluster subset $S_{i j}$ and the arrangement function between the abrasive cluster subset $S_{i j}$ by coordinate transformation according to the coordinate equation of the dimple subset $W_{i j}$ in the workpiece coordinate system and the arrangement function between the dimples. At the same time, as shown in Fig. 2, when the grinding wheel cuts in from point $A_{w 1}$ and cuts out from point $A_{w 2}$ to grind a rectangular dimple, a definite grinding motion interference region is formed between $O_{s 1}$ and $O_{s 2}$ when the grinding wheel moves relative to the workpiece. In this area, the material remaining area is formed due to the effect of the arc length of the grinding wheel. The steep dimple surface perpendicular to the direction of feed speed cannot be generated in the grinding inlet and cut out zones of the grinding 
wheel, which makes it difficult to obtain accurate grinding dimples in accordance with the design dimple by the replay grinding method. However, from the analysis of theoretical research results of structural friction reduction surface, it can be known that the drag reduction or friction reduction performance of dimple surface is related to the geometric shape of the dimple surface, and more importantly, it is related to some feature parameters of its structure [5-8,41-44], which has topological features. Therefore, based on the topology theory, the structured grinding wheel is designed according to the topological features of the dimple surface of the workpiece, and then the topological surface of the workpiece is mapped to meet the use function of the dimple surface of the ground workpiece. Through this grinding method, not only the grinding process of the dimple surface can be simplified, but also the potential of structured grinding wheels for grinding dimple surfaces can be expanded.

\section{Theory of the topological grinding for dimple surface}

\subsection{Establishment of topological space in grinding process}

In the process of dimple grinding as shown in Fig. 1, the Euclidean space of the grinding wheel and the Euclidean space of the workpiece are both measurable spaces, which satisfy the relevant elements of the topological space [40]. Therefore, if the topological space of the grinding wheel is $\boldsymbol{S}_{\boldsymbol{T}}$ and the topological space of the workpiece is $\boldsymbol{W}_{\boldsymbol{T}}$, the grinding process of the dimple surface can be regarded as the homeomorphic mapping process of the grinding wheel space $\boldsymbol{S}_{T}$ to the workpiece space $\boldsymbol{W}_{\boldsymbol{T}}$ [40], namely:

$$
f: S_{T} \rightarrow W_{T}
$$

Where $\boldsymbol{S}_{\boldsymbol{T}}=\left[\cup S_{i j}, i=1,2,3, \ldots, I_{s} . j=1,2,3, \ldots, J_{s}\right]$ is represented as the composition of abrasive cluster subset space, $I_{S}$ and $J_{S}$ are the maximum ordinals of the abrasive cluster structured units in the axial direction and circumferential direction of the wheel. $\boldsymbol{W}_{T}=\left[\cup W_{i j}, i=1,2,3, \ldots, I_{w}, j=1,2,3, \ldots, J_{w}\right]$ is represented as the composition of dimple subset space on workpiece surface, $I_{w}$ and $J_{w}$ are the maximum ordinals of the rows and columns on the workpiece surface corresponding to the arrangement of the abrasive cluster subset. $f=f\left(n_{s}, v_{w}, a_{p}\right)$ is a topological mapping function determined by grinding parameters and grinding wheel geometric parameters. In the grinding process, $S_{i j}$ with convex set features is mapped to an anticonvex set $W_{i j}$ on the workpiece surface with the $1 / n_{s}$ as the cycle. Of course, this is only an abstract expression in the sense of topology, and the actual grinding process is a

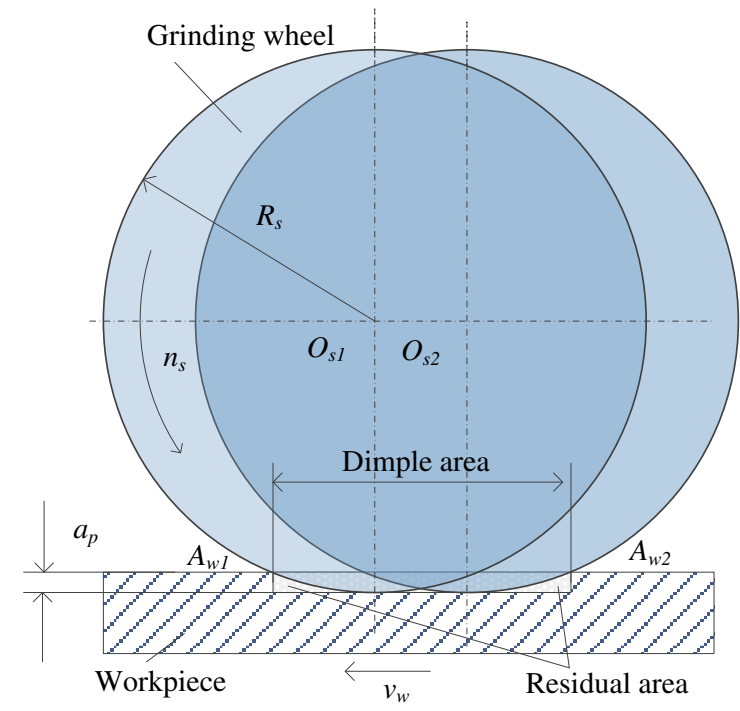

Fig. 2 Schematic diagram of the residual area of dimple grinding 
physical process in which the dimple surface is obtained through the removal of material.

\subsection{Modeling of the topological features of dimple surfaces}

\subsubsection{Modeling of the structured dimple surface}

From the perspective of morphology, structured friction-reducing surface with dimples has a variety of geometric shapes, and each structured dimple surface is formed by dimple elements with specific shapes arranged on the workpiece surface in accordance with certain rules. The shape, size and arrangement of the dimples affect the surface performance for friction-reducing [41-45]. For example, the shape of the dimple has a spherical crown, an ellipsoidal crown, a rectangle, a triangle, etc., and the arrangement of the dimples can be array, staggered, phyllotactic, and random or arrangement following a specific function. In order to facilitate discussion and analysis of issues, taking the array, staggered and phyllotactic arrangement of spherical crown-shaped dimples as an example, the topological feature modeling and analysis are carried out. Fig. 3 shows the Euclidean space parameter description of the three kinds of dimple arrangement surface. The dimple is a spherical crown.

In Fig. 3a, the mathematical description equation of Euclidean space is given by taking the elliptic dimple structure unit as an example. For the convenience of studying the problem, the contour is deformed topologically into ellipsoid crown or spherical crown shape, and the deformation does not overlap, does not produce sharp points, and does not change convex-concave of the contour. Therefore, taking the center of the dimple as the center of the elliptical dimple unit and keeping it consistent with the workpiece coordinate system, the mathematical model of the dimple is as follows:

$$
\left\{\frac{4 x_{w}^{2}}{l_{w}^{2}}+\frac{4 y_{w}^{2}}{w_{w}^{2}} \leq 1\right.
$$

$$
z_{w}=-\frac{h_{w}}{c_{w}}\left[\sqrt{h_{w}^{2}-\frac{4 c_{w}\left(2 h_{w}-c_{w}\right) x_{w}^{2}}{\left(l_{w}\right)^{2}}-\frac{4 c_{w}\left(2 h_{w}-c_{w}\right) y_{w}^{2}}{\left(w_{w}\right)^{2}}}-h_{w}+c_{w}\right]
$$

Where, $c_{w}$ is the radius of the $z_{w}$ axis of the ellipse where the ellipsoid crown is located, and $l_{w}, w_{w}$ and $h_{w}$ are the length, width and depth of the dimple unit respectively. When $l_{w}>w_{w}$, the shape of the dimple unit is elliptic crown. When $l_{w}=w_{w}$, the shape of the dimple unit is spherical crown.

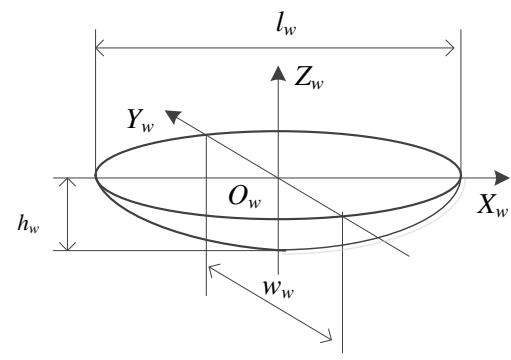

(a) Dimple

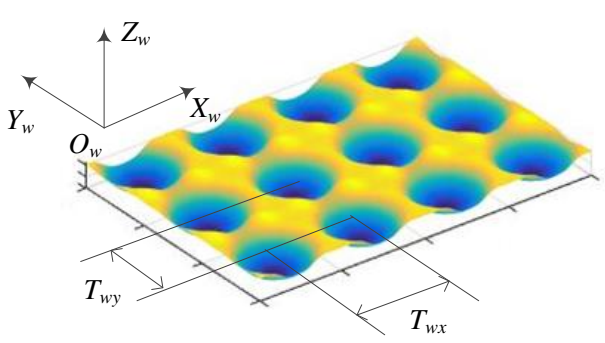

(b) Array pattern

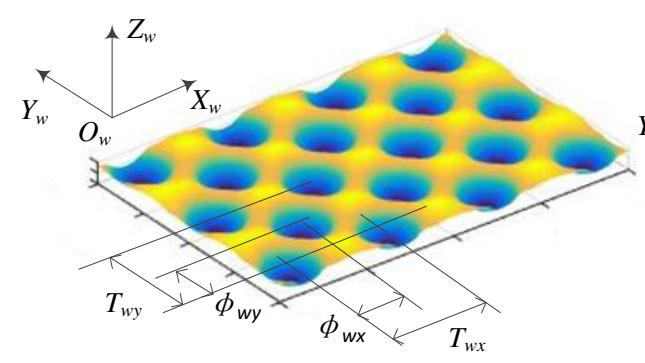

(c) Staggerd pattern

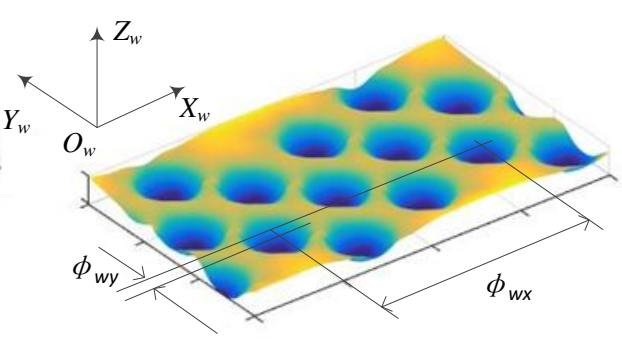

(d) Phyllotactic pattern

Fig. 3 Schematic diagram of the dimple of workpiece surface and its arrangement 
For the three regular dimple arrangements shown in Fig. 2b, Fig. 2c and Fig. 2d, there is a certain arrangement interval and position deviation between adjacent dimple units. Therefore, let the interval between dimple units in the same row or column form the arrangement cycle of the $x_{w}$-axis direction and the $y_{w}$-axis direction as $T_{w x}$ and $T_{w y}$ respectively. The deviation between the positions of different rows and columns results in the phase differences of $\emptyset_{w x}$ and $\emptyset_{w y}$ in the two directions. For the array pattern, The arrangement interval of the structural unit is controlled by the arrangement cycle $T_{w x}$ and $T_{w y}$. The rows and rows are arranged neatly, and the phase difference is $\emptyset_{w x}=\emptyset_{w y}=0$. The cycle of staggered pattern is $T_{w x}$ and $T_{w y}$, respectively. In the case of equal arrangement, the phase difference between even and odd rows in the staggered pattern is $\emptyset_{w x}=T_{w x} / 2$, and the phase difference between even and odd columns is $\emptyset_{w y}=T_{w y} / 2$. The phyllodes arrangement here is the expansion arrangement of the Iterson G Van model [46] based on the cylindrical arrangement with radius $R_{b}$ and growth order $c$, that is, an arrangement on a plane with an expansion length of $2 \pi R_{b}$ [47]. The cycle of expansion is $T_{w x}=$ $T_{w y}=0$, the phase difference $\emptyset_{w x}=2 \pi R_{b}[(i-1) \theta / 360-m+1]$ in the $x_{w}$ direction and the phase difference $\emptyset_{w y}=c(i-1)$ in the $y_{w}$ direction, and each dimple unit is not in the same row and column. Then Eq (1) can be regarded as the basic dimple, and the mathematical equation of the central position of any dimple can be established. The equation is as follows:

(a) Array pattern:

$$
\left\{\begin{array}{r}
x_{w o}^{i j}=(j-1) T_{w x} \\
y_{w o}^{i j}=(i-1) T_{w y} \\
z_{w o}^{i j}=0
\end{array}\right.
$$

(b) Staggered pattern:

$$
\left\{\begin{array}{c}
x_{w o}^{i j}=\frac{(j-1) T_{w x}}{2}, \\
y_{w o}^{i j}=\frac{(i-1) T_{w y}}{2}, \\
z_{w o}^{i j}=0 . \\
i, j \text { are both odd or even. }
\end{array}\right.
$$

(c) Phyllotactic pattern:

$$
\left\{\begin{array}{c}
x_{w o}^{i j}=\emptyset_{w x}=2 \pi R_{b}\left[\frac{(j-1) \theta}{360}-m+1\right], \\
y_{w o}^{i j}=\emptyset_{w y}=c(i-1), \\
z_{w o}^{i j}=0 .
\end{array}\right.
$$

In Eqs. (2), (3) and (4), $i=1,2,3, \cdots, I_{w} . \quad j=1,2,3, \cdots, J_{w}, \quad\left(x_{w o}^{i j} y_{w o}^{i j}, z_{w o}^{i j}\right)$ is the coordinate of the center point of the dimple $W_{i j} . i$ is the ordinal number of the row, and $j$ is the ordinal number of the column. For staggered pattern, $i$ and $j$ must be both odd or both even. For the phyllotactic pattern, the phyllotactic angle $\theta=137.508^{\circ}, i=j=1,2,3, \cdots, I_{w}$, is the ordinal number of structural units. $m$ is the ordinal number that controls the $j$ th structural unit in the $x_{w}$-axis direction, and $\mathrm{m} \leq(j-1) \theta / 360 \leq m+$ $1, m=1,2,3, \ldots, M$ 。

\subsubsection{Establishment of the topological feature vectors of the dimple surface}

According to the topological grinding strategy in Section 1, it is necessary to ensure that the main features of the workpiece surface remain unchanged during the topological grinding process. In order to simplify the problem, for the three kinds of structured dimple surfaces described in equations (2) to (5), only some main parameters are extracted as topological invariants, which are used as topological properties of dimple surfaces. For the point set $W_{i j}$ of the dimple unit, the length $l_{w}$, width $w_{w}$ and depth 
$h_{w}$ of the unit can be used to establish the matrix $\boldsymbol{W}_{\boldsymbol{d}}$ that controls the shape and size of the dimple unit and the attributes of the anticonvex set of the dimple. As for the arrangement rule between units, the arrangement cycle $\left(T_{w x}, T_{w y}\right)$ and the phase difference $\left(\emptyset_{w x}, \emptyset_{w y}\right)$ can be used as the arrangement attribute between control units and the neighborhood attribute of adjacent dimples. Thus, $l_{w}, w_{w}$ and $h_{w}$ are extracted as the topological feature parameters of the structural unit, and $T_{w x}, T_{w y}, \emptyset_{w x}$ and $\emptyset_{w y}$ were taken as the arranged topological feature parameters. The topological feature vector and the arranged topological feature vector of dimple units were established. Topological feature vector is as follows:

(1) Topological feature vector of the dimple unit:

$$
\boldsymbol{W}_{\boldsymbol{d}}=\left[\begin{array}{llll}
\boldsymbol{L}_{\boldsymbol{w}} & \boldsymbol{W}_{\boldsymbol{w}} & \boldsymbol{H}_{\boldsymbol{w}} & 1
\end{array}\right]^{T}
$$

In Eq. (6), $\boldsymbol{L}_{\boldsymbol{w}}, \quad \boldsymbol{W}_{\boldsymbol{w}}$ and $\boldsymbol{H}_{\boldsymbol{w}}$ are the set of feature parameters that describe the length, width and depth of the geometric morphology of the dimple, respectively, and are a set of feature parameters corresponding to the direction of the maximum parameters $l_{w}, w_{w}$, and $h_{w}$, which can reflect the using function. In actual operation, the $X_{w} O_{w} Y_{w}$ coordinate plane and the $X_{w} O_{w} Z_{w}$ coordinate plane are equally spaced and discrete to determine these parameters.

(2) Arranged topological feature vector:

Let the topological feature vector of the dimple arrangement be as follows:

$$
\boldsymbol{W}_{\boldsymbol{a}}=\left[\begin{array}{llll}
T_{w x} & T_{w y} & \emptyset_{w x} & \emptyset_{w y}
\end{array}\right]^{T}
$$

Then the feature parameter vectors corresponding to three specific arrangements are as follows:

(a) array pattern:

(b) Staggered pattern:

$$
\boldsymbol{W}_{\boldsymbol{a} \boldsymbol{a}}=\left[\begin{array}{llll}
T_{w x} & T_{w y} & 0 & 0
\end{array}\right]^{T}
$$

$$
\boldsymbol{W}_{\boldsymbol{a s}}=\left[\begin{array}{llll}
T_{w x} & T_{w y} & \frac{T_{w x}}{2} & \frac{T_{w y}}{2}
\end{array}\right]^{T}
$$

(c) phyllotactic pattern:

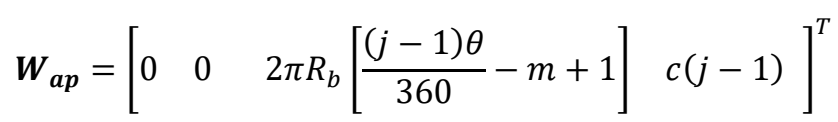

\subsection{Topology analysis and modeling of structured grinding wheel}

\subsubsection{Modeling of the structured surface of grinding wheel}

Fig.3 is the morphology of the structured grinding wheel corresponding to the arrangement of three kinds of abrasive clusters adopted in Fig. 1. In the process of topological mapping grinding, the circumscribed geometric properties and layout properties of the abrasive cluster $S_{\mathrm{ij}}$ on the wheel surface will be topologically mapped along the $X_{w}$-axis of the workpiece coordinate system according to the different grinding parameters. Its radial topological properties will be mapped along the $Z_{w}$ axis of the workpiece according to the different grinding parameters, while the axial properties will remain unchanged and map to the $Y_{w}$ axis direction of the workpiece. For the convenience of discussion, the circumferential arc length, axial width and radial height of the abrasive cluster of the structured grinding wheel are set as $l_{s}, w_{s}$ and $h_{s}$ respectively corresponding to $l_{w}, y_{w}$ and $\mathrm{t} h_{w}$ of the dimple unit. Then the point set of the abrasive cluster $S_{i j}$ will be obtained from the point set of the dimple $W_{\mathrm{ij}}$ on the workpiece surface through topological mapping. Therefore, the equation of abrasive cluster unit on the grinding wheel surface is described as:

$$
f_{s}\left(x_{s}, y_{s}, z_{s}\right)=0, \quad\left(x_{s}, y_{s}, z_{s}\right) \in S_{i j}
$$

Suppose that the arc length arrangement cycle $T_{s l}$ and axial cycle $T_{s y}$ of the abrasive clusters in the grinding wheel with the circle radius $R_{s}$ of the substrate correspond to the arrangement cycle $T_{w x}$ and 
$T_{w y}$ of the dimples on the workpiece surface respectively; The arc length phase difference $\emptyset_{s l}$ and axial phase difference $\emptyset_{s y}$ of the abrasive clusters correspond to the $\emptyset_{w x}$ and $\emptyset_{w y}$ of the dimples on the workpiece surface, respectively; then, for the array pattern of abrasive clusters shown in Fig. 4a, there will be $\emptyset_{s l}=0$ and $\emptyset_{s l}=0$; for the staggered pattern shown in Fig. 4b, there are $\emptyset_{s l}=T_{s l} / 2$ and $\emptyset_{s y}=$ $T_{s y} / 2$; for the phyllotactic pattern shown in Fig. 4c, each circle on the circumference of the grinding wheel and each axial generatrix have only one abrasive cluster unit, and the position of the abrasive cluster on the circumference of the grinding wheel can be described by the phase difference between each other, therefore, according to the Iterson G Van model [46] of the phyllotactic pattern on cylinder surface, $\emptyset_{s l}=(j-1) R_{s} \theta \pi / 180$ and $\emptyset_{s y}=c(i-1)$. Therefore, the position equation of the abrasive cluster is expressed as follows:

(a) Array pattern:

$$
\left\{\begin{array}{c}
x_{s o}^{i j}=R_{S} \cos \left[\frac{(j-1) T_{s x}}{R_{S}}\right], \\
y_{s o}^{i j}=(i-1) T_{s y}, \\
z_{S o}^{i j}=R_{S} \sin \left[\frac{(j-1) T_{S x}}{R_{S}}\right] .
\end{array}\right.
$$

(b) Staggered pattern: 


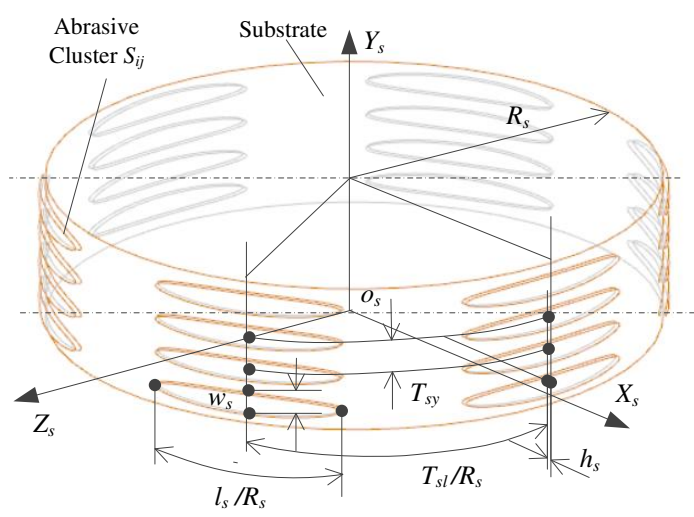

(a) Array pattern

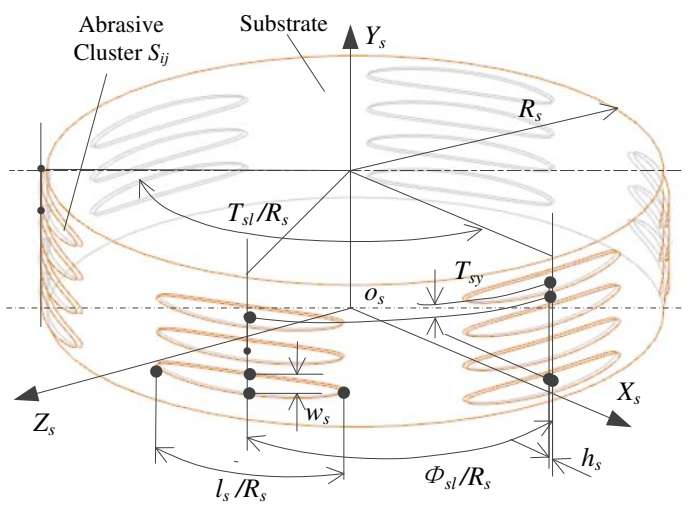

(b) Staggered pattern

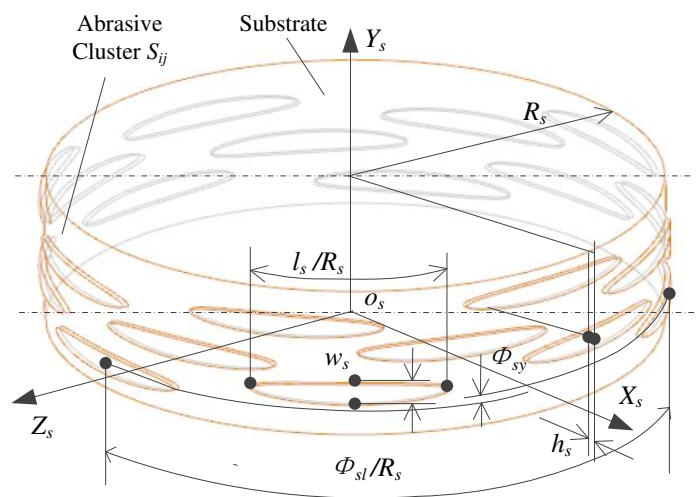

(c) Phyllotactic pattern

Fig.4 Schematic diagram of structured grinding wheel

(c) Phyllotactic pattern:

$$
\left\{\begin{array}{c}
x_{s o}^{i j}=R_{s} \cos \left[\frac{(j-1) T_{s x}}{2 R_{s}}\right] \\
y_{s o}^{i j}=\frac{(i-1) T_{s y}}{2} \\
z_{s o}^{i j}=R_{s} \sin \left[\frac{(j-1) T_{s x}}{2 R_{S}}\right] .
\end{array}\right.
$$

$$
\left\{\begin{array}{r}
x_{s o}^{i j}=R_{s} \cos \left(\frac{\emptyset_{s l}}{R_{S}}\right)=R_{S} \cos \left[\frac{(j-1) \theta \pi}{180}\right], \\
y_{s o}^{i j}=\emptyset_{s y}=c(i-1), \\
z_{s o}^{i j}=R_{S} \sin \left(\frac{\emptyset_{s l}}{R_{S}}\right)=R_{S} \sin \left[\frac{\theta(j-1) \pi}{180}\right] .
\end{array}\right.
$$

In Eqs. (12) to (14), $i=1,2, \cdots, I_{s}, j=1,2, \cdots, J_{s} . I_{s}$ is the axial ranking number of abrasive clusters on the 
wheel surface; $J_{s}$ is the number of circumferential rows of abrasive clusters. For staggered pattern, $i$ and $j$ must be odd or even, at the same time. For phyllotactic pattern, $i=j=1,2, \cdots, I_{s}, I_{s}=J_{s}$. $\left(x_{s o}^{i j}, y_{s o}^{i j}, z_{s o}^{i j}\right)$ is the position coordinates of the center of the abrasive cluster.

\subsubsection{Establishment of the topological feature vectors of grinding wheel}

During the grinding process, the topological features of the grinding wheel surface are correspondingly mapped to the workpiece surface. Based on the above analysis of the abrasive clusters of the grinding wheel and the established equations, the geometric and arrangement topological parameters of the abrasive clusters can be extracted, and the topological feature vector $\boldsymbol{S}_{\boldsymbol{d}}$ of the abrasive clusters on the wheel surface and the topological feature vector $\boldsymbol{S}_{\boldsymbol{a}}$ of its arrangement can be established, as follows:

(1) Topological feature vector of abrasive cluster unit

$$
\boldsymbol{S}_{\boldsymbol{d}}=\left[\begin{array}{llll}
\boldsymbol{L}_{\boldsymbol{s}} & \boldsymbol{W}_{\boldsymbol{s}} & \boldsymbol{H}_{\boldsymbol{s}} & 1
\end{array}\right]^{T}
$$

In Eq. (15), $\boldsymbol{L}_{\boldsymbol{s}}$ is the set of feature arc lengths of the abrasive clusters along the cylindrical surface of the wheel substrate. $\boldsymbol{W}_{\boldsymbol{s}}$ is the set of the feature widths of the abrasive clusters along the axial direction of the cylindrical surface, and $\boldsymbol{H}_{\boldsymbol{s}}$ is the set of the feature heights of the abrasive clusters relative to the normal direction of the cylindrical surface. $\boldsymbol{L}_{\boldsymbol{s}}, \boldsymbol{W}_{\mathrm{s}}$ and $\boldsymbol{H}_{\boldsymbol{s}}$ correspond to $\boldsymbol{L}_{\boldsymbol{w}}, \boldsymbol{W}_{\boldsymbol{w}}$ and $\boldsymbol{H}_{w}$ of the dimple unit on the workpiece surface.

(2) Topological feature vector of the arrangement of abrasive clusters

Suppose the feature vector of the arrangement of abrasive clusters is:

$$
\boldsymbol{S}_{\boldsymbol{a}}=\left[\begin{array}{llll}
T_{s l} & T_{s y} & \emptyset_{s l} & \emptyset_{s y}
\end{array}\right]^{T}
$$

Then, the topological feature vectors of the three abrasive clusters corresponding to Eq. (16) are as follows:

(a) Array pattern:

$$
\boldsymbol{S}_{\boldsymbol{a} \boldsymbol{a}}=\left[\begin{array}{llll}
T_{s l} & T_{s y} & 0 & 0
\end{array}\right]^{T}
$$

(b) Staggered pattern:

$$
\boldsymbol{S}_{a s}=\left[\begin{array}{llll}
T_{s l} & T_{s y} & \frac{T_{s l}}{2} & \frac{T_{s y}}{2}
\end{array}\right]^{T}
$$

(c) Phyllotactic pattern:

$$
\boldsymbol{S}_{a p}=\left[\begin{array}{llll}
T_{s l} & T_{s y} & \frac{(j-1) R_{s} \theta \pi}{180} & c(i-1)
\end{array}\right]^{T}
$$

\subsection{Topological mapping matrix of grinding dimple process}

(1) Analysis of the mapping process of generating dimple surface

In the process of grinding the surface of the dimple, the structured grinding wheel grinds the surface of the workpiece by an up-grinding method. The abrasive particles of the abrasive cluster make an extended epicycloid motion relative to the workpiece, and the cutting sequence of each abrasive grain in the circumferential direction of the grinding wheel It is different, and its radial protrusion height is also different, so it produces overlapping trajectories in the cross-section that interferes with the workpiece, removes material through the envelope [48], and generates a dimple geometry. Fig. 5 shows the motion trajectory diagram of the enveloping dimples in the $X_{w}$ direction of a plurality of abrasive grains on two adjacent abrasive clusters. It is not difficult to know from Fig. 1 that in the process of mapping dimples from the abrasive cluster topology, no relative movement occurs between the $Y_{s}$ direction of the grinding wheel and the $Y_{w}$ direction of the workpiece. Therefore, the geometric mapping between the topology feature width $\boldsymbol{W}_{\boldsymbol{s}}$ of the abrasive cluster and the topology feature width $\boldsymbol{W}_{\boldsymbol{w}}$ of the dimple maintain an 
identical. At the same time, the geometric mapping between the arrangement features $T_{s}$ and $\emptyset_{s y}$ of the abrasive cluster and the arrangement features $T_{w}$ and $\emptyset_{s w}$ of the dimples will also remain identical. For the abrasive cluster with convex set feature on the grinding wheel surface, the radial height feature $\boldsymbol{H}_{\boldsymbol{s}}$ of the abrasive cluster on the substrate surface can be set to maintain an identical geometric mapping with the topological feature depth $\boldsymbol{H}_{\boldsymbol{w}}$ of the anti-convex set dimple on the workpiece surface in the direction of $Z_{w}$. Therefore, during the grinding process, the mapping change is between the feature length $\boldsymbol{L}_{\boldsymbol{s}}$ of the abrasive cluster and the feature length $\boldsymbol{L}_{\boldsymbol{w}}$ of the dimple in the $X_{\mathrm{w}}$ axis direction under the conditions of different grinding parameters, and between the arrangement parameters $T_{l s} 、 \emptyset_{l s}$ and $T_{w x} 、 \emptyset_{w x}$.

Fig. 6 shows a schematic diagram of grinding the dimples in the direction of the center section of the abrasive cluster. In Fig. 6, when the grinding wheel moves from the $\mathrm{O}_{s 1}$ position to the $O_{s 2}$ position, the abrasive cluster completed the generating of the first dimple, and when it moves to the $O_{s 3}$ position, the

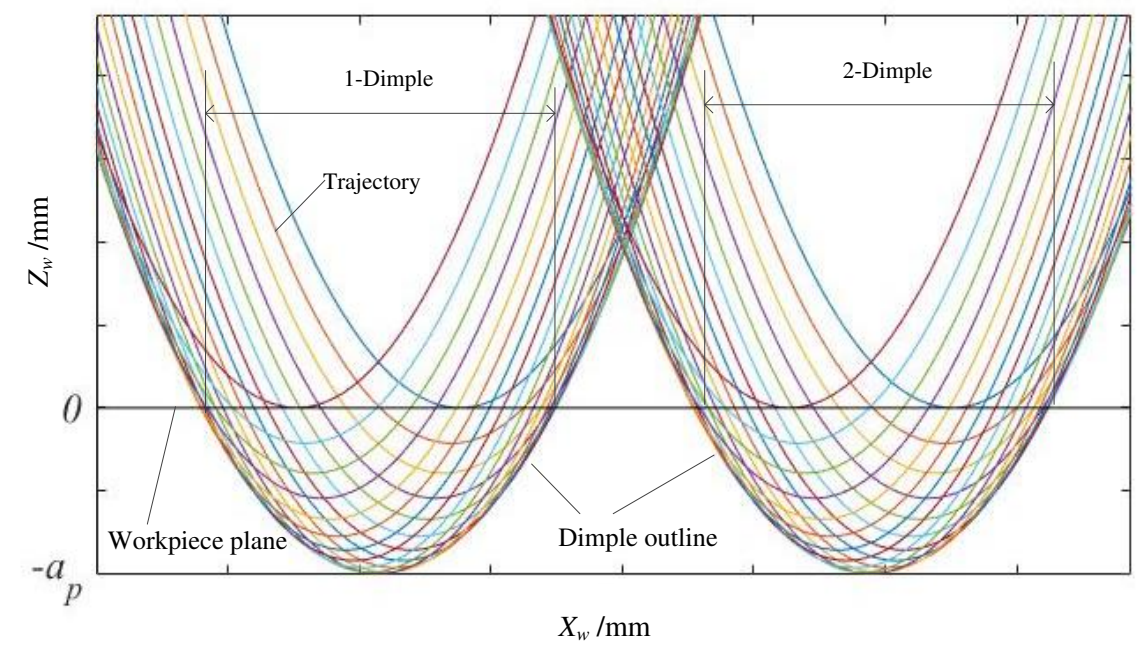

Fig. 5 Schematic diagram of the trajectory of the cross-section of two adjacent abrasive clusters

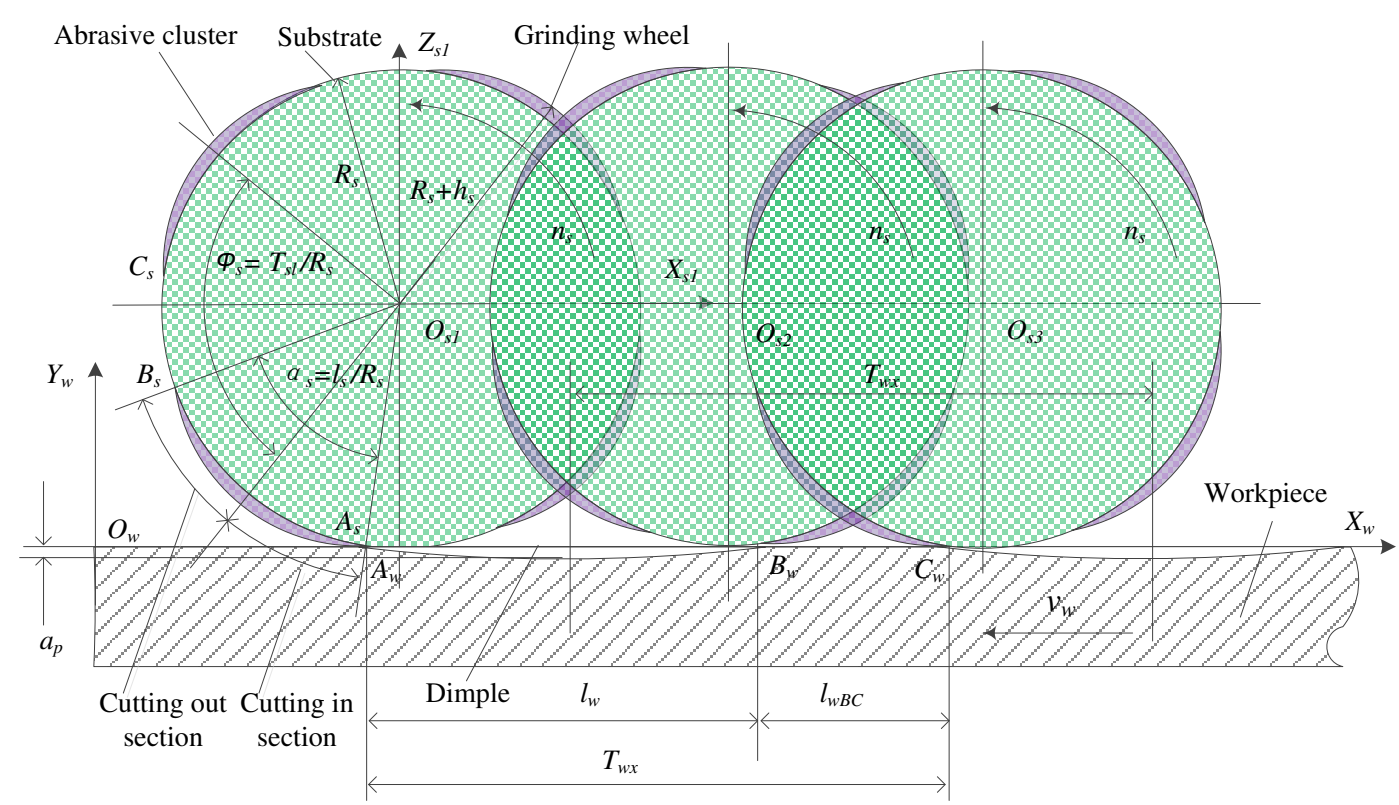

Fig. 6 Schematic diagram of the grinding process of dimple cross-section

subsequent abrasive clusters will generate the second dimple. In this process, the abrasive cluster $\boldsymbol{A}_{\boldsymbol{s}}$ point on the $\widehat{A_{s} B_{S}}\left(=l_{s}\right)$ segment of the abrasive cluster is cut in from the $A_{w}$ point on the surface of the 
workpiece, and the $B_{s}$ point is cut out from the $B_{w}$ point of the workpiece, and a dimple unit $\overline{A_{w} B_{w}}\left(=l_{w}\right)$ is ground. The $\widehat{B_{S} C_{s}}$ section of the grinding wheel surface has no abrasive contact with the workpiece, and forms the $\overline{B_{w} C_{w}}$ section corresponding to the workpiece surface, which becomes the platform-shaped separation interval between the dimple units, and the interval length is $l_{w B C}$. When $l_{w B C} \geq 0$, a dimpled surface is grinded on the workpiece surface. In fact, it can be inferred from Fig. 6 that adjacent dimple elements intersect when $T_{w x}<l_{w}$; the dimple units are connected when $T_{w x}=l_{w}$; the dimple units are separated and a dimple-type structured surface can be generated when $T_{w x}>l_{w}$. Therefore, based on the grinding kinematics relationship, when the abrasive clusters of the grinding wheel rotate for one cycle time, that is, $\Phi_{s} / 2 \pi n_{s}=T_{s l} / 2 \pi n_{s} R_{s}$, the workpiece will be fed with $v_{w}$ for an arrangement cycle $T_{w x}$, the relationship between the arrangement cycles $T_{s l}$ and $T_{w x}$ is as follows:

$$
T_{w x}=\frac{\varphi_{s}}{2 \pi n_{s}} \times v_{w}=\frac{T_{s l}}{2 \pi R_{s}} \times \frac{v_{w}}{n_{s}}
$$

In Eq. (20), $P_{s}=2 \pi / N$ is the center angle of adjacent abrasive clusters, and $N$ is the largest ordinal number of abrasive clusters arranged on the same circumference of the grinding wheel. For array and staggered pattern, $N=J_{s}$; for phyllotactic pattern, $N=1$.

Similarly, since the geometric position relationship between the arrangement cycle $T_{s l}$ and the phase difference $\emptyset_{l s}$ is fixed, the following relationship also exists between the phase difference $\emptyset_{l s}$ and $\emptyset_{w x}$ :

$$
\emptyset_{w x}=\frac{\varphi_{S}}{2 \pi n_{s}} \times v_{w}=\frac{\emptyset_{s l}}{2 \pi R_{s}} \times \frac{v_{w}}{n_{s}}
$$

In the actual design of the radial geometry of the abrasive cluster on the surface of the grinding wheel base, it is necessary to ensure that the radial height growth rate of the inlet section and the attenuation rate of the cutting section of the abrasive cluster as shown in Fig. 6 can enable continuous progressive grinding when grinding the dimples on the workpiece surface, without rapidly increasing the grinding depth. In this process, the generating length $l_{w}$ of the dimple in the $X_{w}$ direction is the combined effect of the geometric interference chord length of the grinding wheel under the condition of the grinding depth $a_{p}$ of the abrasive cluster and the movement length of the workpiece during the cutting-in and cutting-out time of the abrasive cluster[49]. However, due to the different radii of the abrasives on the abrasive clusters relative to the center of the grinding wheel, the chord length of the interference changes, and the chord length increases with the change of feed time is also non-linear. Therefore, it can be approximately simplified as the generating length of the dimple is composed of the interference chord length at the average grinding depth $\overline{a_{p}}$ and the average feed motion length in the $X_{w}$ direction when the grinding wheel rotates through the abrasive cluster area, namely:

$$
l_{w} \approx \frac{l_{s}}{2 \pi R_{s}} \times \frac{v_{w}}{n_{s}}+2 \sqrt{2 \overline{R_{s}} \overline{a_{p}}}
$$

In Eq.(22), $\overline{R_{s}}$ is the average value of the radial radius of the abrasive clusters corresponding to the $l_{\mathrm{s}}$ section.is the average value of the abrasive cluster radius. In the grinding mapping process, from the perspective of the grinding depth identity mapping, $a_{p}=h_{s}=h_{w}$ can be determined. Under the condition that the feature parameter of the depth of the dimple is directly mapped along the radial direction of the grinding wheel, the values of $\overline{R_{s}}$ and $\overline{a_{p}}$ can be calculated.

(2) Topological mapping matrix between workpiece and grinding wheel

According to the above analysis of the generating process of the maximum feature size and arrangement of the dimples, it can be seen that the topological corresponding relationship between the dimple and the abrasive cluster is equivalent transformation in the width and depth directions, while the topological deformation of stretching and bending occurs in the length direction due to the influences of 
the contour shape of the abrasive cluster and grinding parameters. Moreover, there is one - to - one mapping relationship between the set points of the dimple and the set points of the abrasive cluster as well as the arrangement relation, which forms a topological mapping relationship. Therefore, according to equations (20), (21) and (22) and the analysis results of the mapping relationship of the dimples in different directions, the size topological mapping matrix $\boldsymbol{F}_{\boldsymbol{d}}$ and the arrangement topological mapping matrix $\boldsymbol{F}_{\boldsymbol{a}}$ in the grinding process can be established as follows:

(a) Topological mapping matrix of the size features:

$$
\boldsymbol{F}_{\boldsymbol{d}}=\left[\begin{array}{cccc}
\frac{v_{w}}{2 \pi R_{s} n_{s}} & 0 & 0 & 2 \sqrt{2 \overline{R_{s}^{s} a_{p}^{s}}} \\
0 & 1 & 0 & 0 \\
0 & 0 & 1 & 0 \\
0 & 0 & 0 & 1
\end{array}\right]
$$

Where $\overline{R_{S}^{s}}$ is the average radius in the area corresponding to the length of the $s$-th divided section of the abrasive cluster, $\overline{a_{p}^{s}}$ is the average grinding depth of the $s$-th divided section. When the abrasive cluster is the maximum length $l_{\mathrm{s}}, \overline{R_{s}^{s}}=\overline{R_{s}}$ and $\overline{a_{p}^{s}}=\overline{a_{p}}$.

(b) Topological mapping matrix of the arrangement features:

$$
\boldsymbol{F}_{\boldsymbol{a}}=\left[\begin{array}{cccc}
\frac{v_{w}}{2 \pi R_{s} n_{s}} & 0 & 0 & 0 \\
0 & 1 & 0 & 0 \\
0 & 0 & \frac{v_{w}}{2 \pi R_{s} n_{s}} & 0 \\
0 & 0 & 0 & 1
\end{array}\right]
$$

Therefore, based on equations (6), (7), (15), (16) and (23), (24), the topological mapping equations in the grinding process can be obtained as follows:

$$
\left\{\begin{array}{l}
W_{d}=F_{d} S_{d} \\
W_{a}=F_{a} S_{a}
\end{array}\right.
$$

Similarly, if the mapping of the grinding process is regarded as an identity mapping, for the convenience of designing the grinding wheel, the corresponding inverse mapping relationship from the workpiece to the grinding wheel can be given:

$$
\left\{\begin{array}{l}
S_{d}=F_{d}^{-1} W_{d} \\
S_{a}=F_{a}^{-1} W_{a}
\end{array}\right.
$$

Among them, $\boldsymbol{F}_{\boldsymbol{d}}{ }^{-1}$ and $\boldsymbol{F}_{\boldsymbol{a}}{ }^{-1}$ are as follows:

$$
\begin{gathered}
\boldsymbol{F}_{\boldsymbol{d}}{ }^{-1}=\left[\begin{array}{cccc}
\frac{2 \pi R_{s} n_{\mathrm{s}}}{v_{\mathrm{w}}} & 0 & 0 & -\frac{4 \pi R_{s} n_{s}}{v_{w}} \sqrt{2 \overline{R_{s}^{S}} a_{p}^{s}} \\
0 & 1 & 0 & 0 \\
0 & 0 & 1 & 0 \\
0 & 0 & 0 & 1
\end{array}\right] \\
\boldsymbol{F}_{\boldsymbol{a}}{ }^{-1}=\left[\begin{array}{rrcc}
\frac{2 \pi R_{s} n_{s}}{v_{w}} & 0 & 0 & 0 \\
0 & 1 & 0 & 0 \\
0 & 0 & \frac{2 \pi R_{s} n_{s}}{v_{w}} & 0 \\
0 & 0 & 0 & 1
\end{array}\right]
\end{gathered}
$$

\section{Design and manufacturing of structured grinding wheel}

\subsection{Design of the grinding wheel}

In the actual design of the grinding wheel, the substrate radius $R_{s}$ and the configuration form of the grinding wheel can be given first, as well as the number of circumscribed columns $J_{s}$, so that the circumscribed $T_{s l}$ and $\emptyset_{s l}$ of the grinding wheel can also be determined first. Then, according to $T_{w x}, \emptyset_{w x}$, $T_{w y}$ and $\emptyset_{w y}$ known to the workpiece,

$T_{s y}, \emptyset_{s y}$ and $v_{w} / n_{s}$ can be determined by using the configuration equations in Eqs. (25) and (26). Then, 
according to the feature quantities $\boldsymbol{L}_{\boldsymbol{w}}, \boldsymbol{W}_{\boldsymbol{w}}$ and $\boldsymbol{H}_{\boldsymbol{w}}$ of the dimples, $\boldsymbol{L}_{\boldsymbol{s}}, \boldsymbol{W}_{s}$ and $\boldsymbol{H}_{\boldsymbol{s}}$ are obtained by using the size equations of the dimples in Eqs. (25) and (26).

In the design of the abrasive cluster of the grinding wheel, firstly, the dimple unit shown in Fig. 2a is discretely divided along the equal spacing of $X_{w}$ and $Y_{w}$ directions to determine the feature parameter set of length, and at the same time, the depth feature set of $Z_{w}$ direction is determined. Then, the cylinder surface of the grinding wheel is correspondingly divided in the topological space of the grinding wheel. When the abrasive cluster unit is divided, the axial discrete segmentation corresponds to the $Y_{w}$ direction division of the workpiece dimple, the circumferential segmentation with discrete equal angle corresponds to the $X_{w}$ direction division of the workpiece dimple, and then the peripheral size set $\boldsymbol{L}_{\boldsymbol{s}}$, axial size set $\boldsymbol{W}_{\boldsymbol{s}}$, and the radial size set $\boldsymbol{H}_{\boldsymbol{s}}$ can be obtainand. Finally, the feature dimensions of $\boldsymbol{L}_{\boldsymbol{s}} 、 \boldsymbol{W}_{\boldsymbol{s}}$ and $\boldsymbol{H}_{\boldsymbol{s}}$ are calculated by using the size mapping equation of abrasive cluster in Eq. (26).

According to the above method, under the condition of the known dimple size as shown in Tab. 1, the arrangement and size parameters of the corresponding abrasive clusters are designed, and the digital

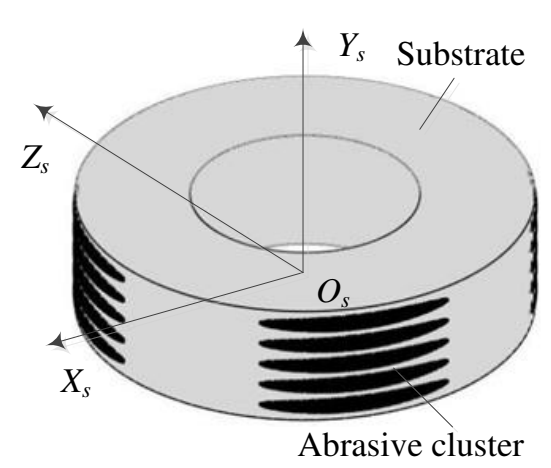

(a) Array pattern

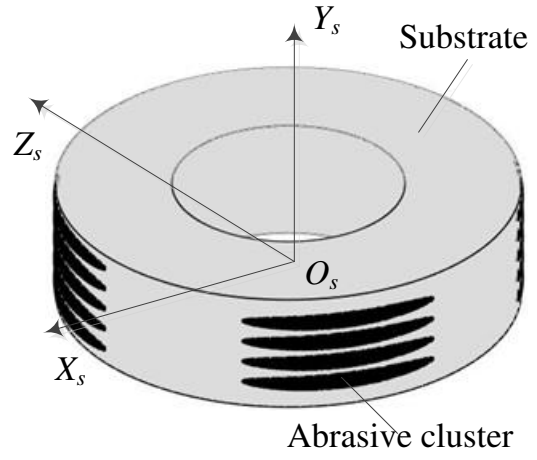

(a) Staggered pattern

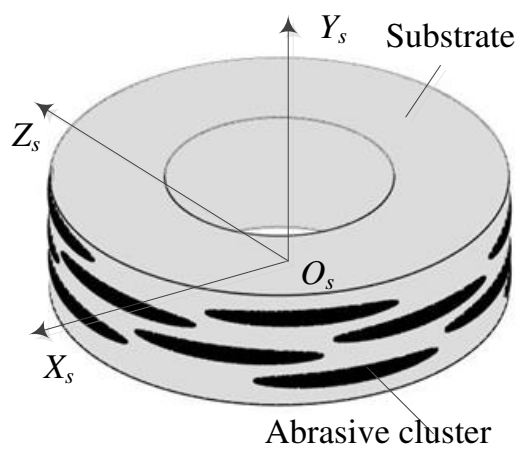

(a) Phyllotactc pattern

Fig.7 Digital grinding wheel with different arrangement of abrasive clusters

grinding wheels with the array, staggered and phyllotactic pattern of the abrasive clusters shown in Fig. 7 are designed by using software.

Tab. 1. Topological design parameters of workpiece and grinding wheel

\begin{tabular}{|c|c|c|c|c|c|c|}
\hline \multirow[t]{2}{*}{ Parameters } & \multicolumn{3}{|c|}{ Workerpiece（known） } & \multicolumn{3}{|c|}{ Wheel: Diameter $\Phi 60 \mathrm{~mm}$, Thickness } \\
\hline & $\begin{array}{l}\text { Array } \\
\text { pattern }\end{array}$ & $\begin{array}{l}\text { Staggered } \\
\text { pattern }\end{array}$ & $\begin{array}{l}\text { Phyllotactic } \\
\text { pattern }\end{array}$ & $\begin{array}{l}15 \mathrm{~mm} . \\
\text { Array } \\
\text { pattern }\end{array}$ & $\begin{array}{l}\text { Staggered } \\
\text { pattern }\end{array}$ & $\begin{array}{l}\text { Phyllotactic } \\
\text { pattern }\end{array}$ \\
\hline Unit sizes, mm & $\begin{array}{l}l_{w}=3 \\
w_{w}=2 \\
h_{w}=0.020\end{array}$ & $\begin{array}{l}l_{w}=3 \\
w_{w}=2 \\
h_{w}=0.020\end{array}$ & $\begin{array}{l}l_{w}=3 \\
w_{w}=2 \\
h_{w}=0.020\end{array}$ & $\begin{array}{l}l_{s}=26 \\
w_{s}=2 \\
h_{s}=0.020\end{array}$ & $\begin{array}{l}l_{s}=26 \\
w_{s}=2 \\
h_{s}=0.020\end{array}$ & $\begin{array}{l}l_{s}=26 \\
w_{s}=2 \\
h_{s}=0.020\end{array}$ \\
\hline $\begin{array}{l}\text { Unit } \\
\text { arrangement } \\
\mathrm{mm} \quad v_{w} / n_{s}= \\
\left.13, a_{p}=20 \mu \mathrm{m}\right)\end{array}$ & $\begin{array}{l}T_{w x}=3.25 \\
T_{w y}=3 \\
\emptyset_{w x}=0 \\
\emptyset_{w y}=0\end{array}$ & $\begin{array}{l}T_{w x}=6.5 \\
T_{w y}=3 \\
\emptyset_{w x}=3.25 \\
\emptyset_{w y}=1.5\end{array}$ & $\begin{aligned} T_{w x} & =0 \\
T_{w y} & =0 \\
\varnothing_{\mathrm{wx}} & =2 \Pi R_{b}\left[\frac{(\mathrm{j}-1) \theta}{360}-\mathrm{m}+1\right] \\
\emptyset_{w y} & =0.7(i-1)\end{aligned}$ & $\begin{array}{l}T_{s l}=47.1 \\
T_{s y}=3 \\
\emptyset_{s l}=0 \\
\emptyset_{s y}=0\end{array}$ & $\begin{array}{l}T_{s l}=94.2 \\
T_{s y}=3 \\
\emptyset_{s l}=47.1 \\
\emptyset_{s y}=1.5\end{array}$ & $\begin{array}{l}T_{s l}=0 \\
T_{s y}=0 \\
\varnothing_{\mathrm{sl}}=71.96(\mathrm{j}-1) \\
\emptyset_{s y}=0.7(i-1)\end{array}$ \\
\hline $\begin{array}{l}\text { Rows and } \\
\text { columns }\end{array}$ & $\begin{array}{l}I_{w}=5 \\
j=1,2,3, \ldots, J_{w}\end{array}$ & $\begin{array}{l}I_{w}=9 \\
j=1,2,3, . ., J_{w}\end{array}$ & $I_{w}=J_{w}=18$ & $\begin{array}{l}I_{s}=5 \\
J_{s}=4\end{array}$ & $\begin{array}{l}I_{s}=9 \\
J_{s}=4\end{array}$ & $i=j=1,2,3, . ., 18$ \\
\hline
\end{tabular}

\subsection{Manufacture of the grinding wheel}

In the manufacturing process of the grinding wheel, a combination of masking, corrosion and electroplating is used [50,51], and the process flow is shown in Fig. 8. Firstly, the mask is designed and manufactured according to the structure of the grinding wheel shown in Fig. 7 and the parameters in Tab.

1. Then lithography and corrosion were carried out on the substrate of the grinding wheel to form a 
convex hull arranged in order on the substrate surface of the grinding wheel, and the convex hull was precisely shaped to achieve the designed geometric shape. When dressing the geometry of the convex hull, the amount of pre-dressing should be given based on the average diameter of the abrasive size. Finally, the consolidating abrasive grains and thickening coating on the convex hull by electroplating form a grinding wheel to ensure that the contour height of the abrasive clusters meets the design requirements. In order to improve the mechanical properties of the electroplated layer, the electroplated grinding wheel was placed in a thermostat for hydrogen evolution and stress relief. The substrate diameter of the electroplated grinding wheel is $\Phi 60 \mathrm{~mm}$, and the axial thickness is $15 \mathrm{~mm}$. The abrasive used is CBN abrasive with 140/150 mesh. The electroplating solution is a bright nickel plating solution with $\mathrm{NiSO}_{2}$ as the main salt [50, 51]. Fig. 9 shows an electroplated grinding wheel with an array, staggered and phyllotactic pattern of the abrasive clusters.

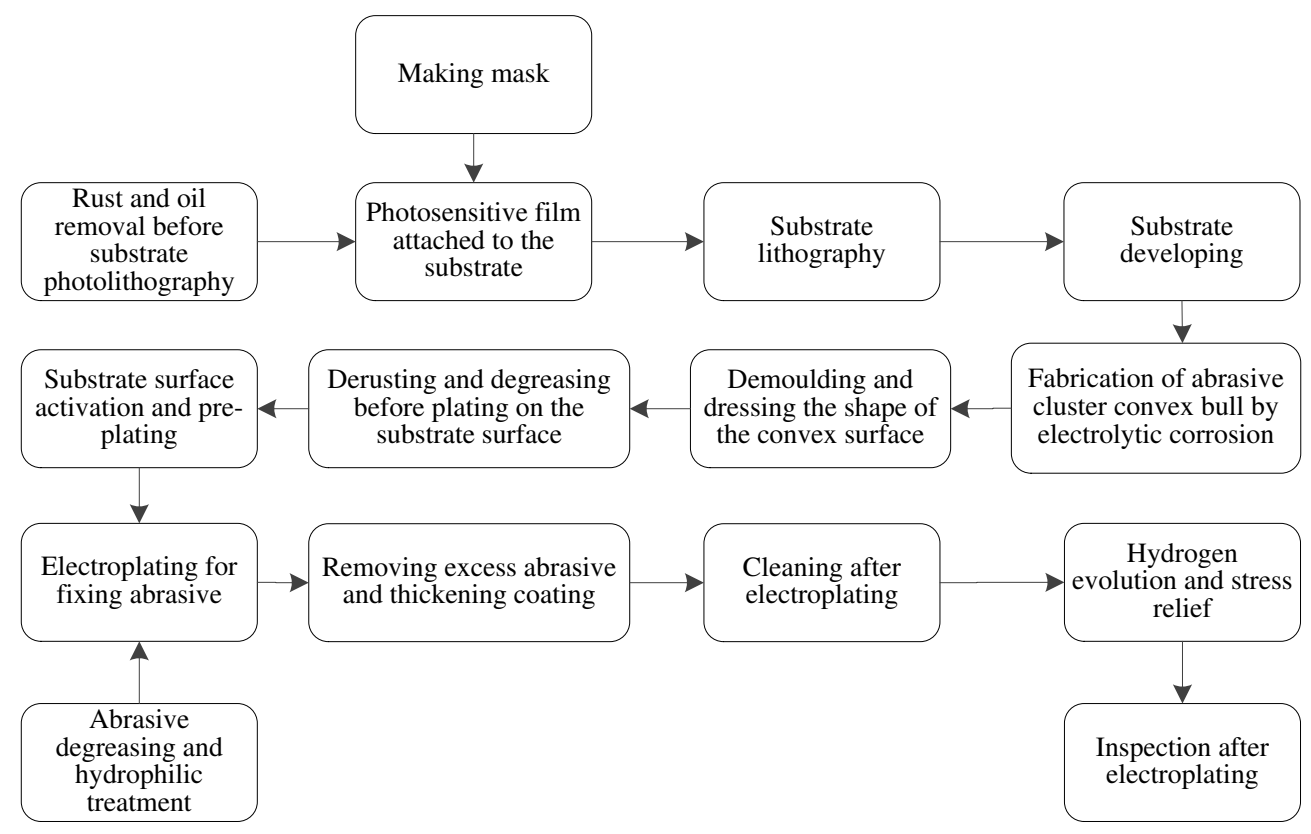

Fig . 8 Process flow chart of electroplating grinding wheel

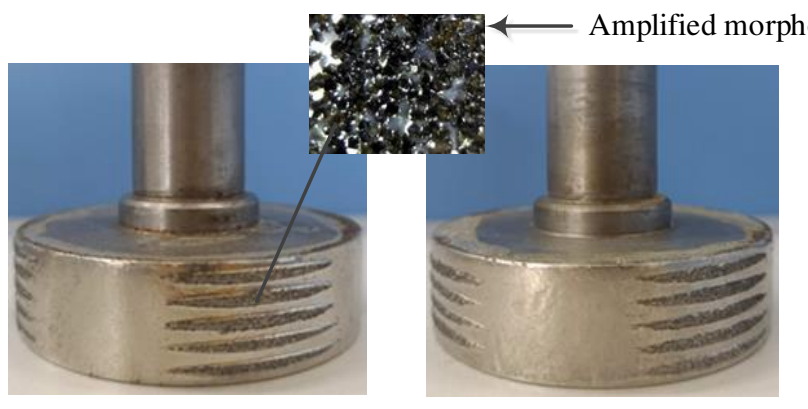

(a) Array pattern

(b) Staggered pattern

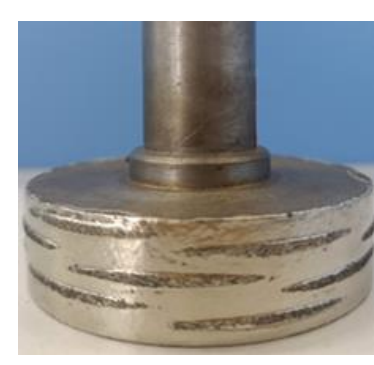

(c) Phyllotactic pattern

Fig9. Electroplated CBN grinding wheel with ordered abrasive clusters

\section{Geometric simulation of grinding dimple surface}

\subsection{Simulation strategy and conditions}

According to the topological feature parameters of Tab. 1 and the digital grinding wheel shown in Fig.

7 , the geometric simulation of generating the dimple surface during the grinding process was carried out 
using computer software programming. The simulation flow chart is shown in Fig.10. In the simulation, the distance between the grinding wheel and the workpiece surface as shown in Fig. 1 is set as $e=R_{S}+$ $h_{s}-a_{p}$. In the simulation, the position of the abrasive grain relative to the trajectory point of the workpiece surface is calculated according to the trajectory equation of the surface grinding [48], and then the grinding interference state between the abrasive particle and the workpiece is judged to determine the surface topography of the workpiece. CBN abrasive size is 140/150 Mesh, and its average diameter is set to $90 \mu \mathrm{m}$. Only the macroscopic contour of the dimple is considered and the microscopic roughness effect is ignored.

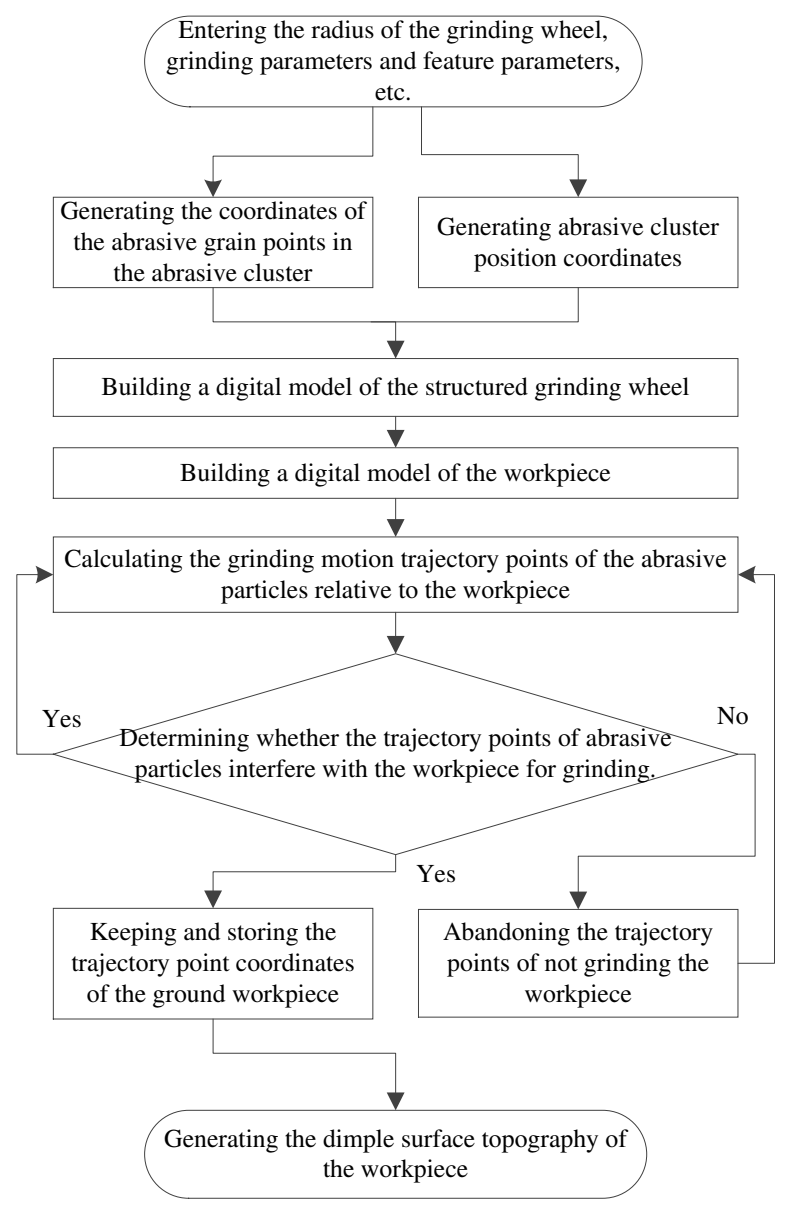

Fig. 10 Simulation flow chart for grinding dimple surface

\subsection{Simulation results and analysis}

Fig. 11 is the dimple surface with array, staggered and phyllotactic pattern realized by simulation under the designed parameters of Tab.1. And at this time, the dimples of array, staggered and phyllotactic pattern all form a structure in a separated state. The average cycle of the array pattern in the $X_{w}$ direction is $3.25 \mathrm{~mm}$, and the average cycle in the $Y_{w}$ direction is $3 \mathrm{~mm}$. The average cycle of the staggered pattern in the $X_{w}$ direction is $6.5 \mathrm{~mm}$, and the average phase difference is $3.25 \mathrm{~mm}$; the average cycle in the $Y_{w}$ direction is $3 \mathrm{~mm}$, and the phase difference is $1.5 \mathrm{~mm}$. The initial phase difference in the $X_{w}$ direction of the phyllotactic pattern is $4.96 \mathrm{~mm}$, and the initial average phase difference in the $Y_{w}$ direction is $0.7 \mathrm{~mm}$. The maximum length, width and depth of the dimples are also consistent with the design dimensions. It shows that the topological grinding method can realize the diversification of dimple surface. 
In order to obtain the mapping effect of $v_{w} / n_{s}$ on the dimple of the workpiece surface, on the basis of the structured grinding wheel designed based on $v_{w} / n_{s}=13$ in Tab. 1 , the structured surface morphology of dimples arranged in array was simulated under the conditions of $v_{w} / n_{s}=9,12,14$ and 15 respectively, as shown in Fig.12. When $v_{w} / n_{s}=9$, adjacent dimple units intersect in the $X_{w}$ direction. When $v_{w} / n_{s}=12,14$ and 15, with the increase of $v_{w} / n_{s}$, the maximum length of dimple unit in the $X_{w}$ direction is $2.9 \mathrm{~mm}, 3.09 \mathrm{~mm}$ and $3.20 \mathrm{~mm}$, respectively, showing an increasing trend, while the width in the $Y_{w}$ direction remains the same as the design width of $2 \mathrm{~mm}$, and the maximum depth remains $20 \mu \mathrm{m}$; Similarly, the arrangement cycle in the $X_{w}$ direction is $3 \mathrm{~mm}, 3.5 \mathrm{~mm}$, and $3.75 \mathrm{~mm}$ respectively, showing an increasing trend, and the cycle in the $Y_{w}$ direction also remains unchanged at the design cycle of $3 \mathrm{~mm}$. The simulation results verify the consequences in the design analysis that as the speed ratio further increases, the depth and width of the dimple unit remain unchanged, and the length and cycle of the structural unit increase.

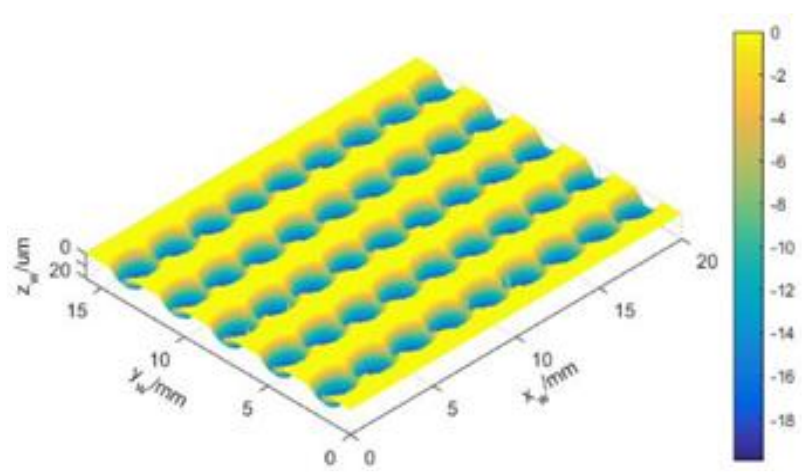

(a) at $v_{w} / n_{s}=9, n_{s}=1800 \mathrm{r} / \mathrm{min}$ and $v_{w}=16200 \mathrm{~mm} /$ $\min$.

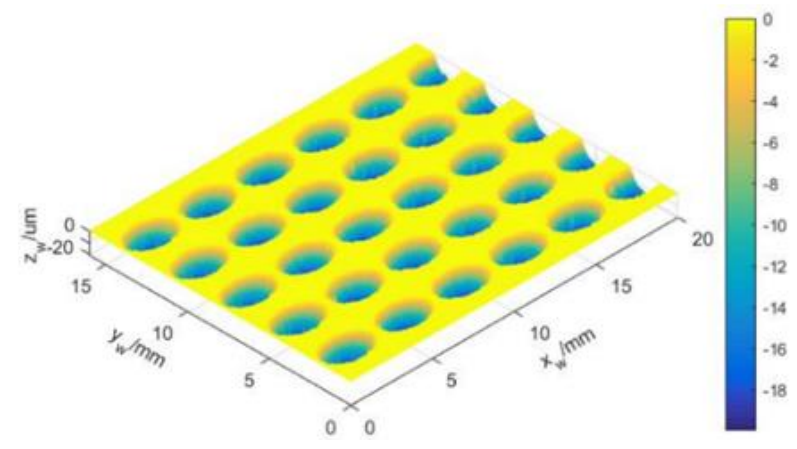

(c) at $v_{w} / n_{s}=14, n_{s}=1800 \mathrm{r} / \mathrm{min}$ and $v_{w}=25200 \mathrm{~mm} /$ $\min$.

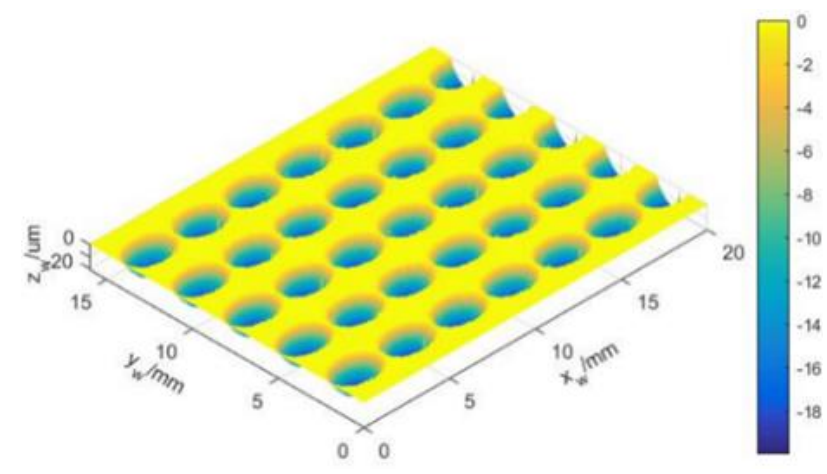

(b) at $v_{w} / n_{s}=12, n_{s}=1800 \mathrm{r} / \mathrm{min}$ and $v_{w}=21600 \mathrm{~mm} /$ $\min$.

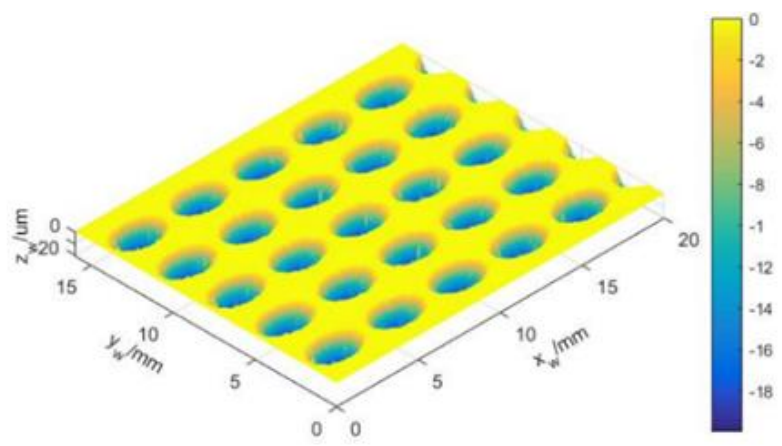

(d) at $v_{w} / n_{s}=15, n_{s}=1800 \mathrm{r} / \mathrm{min}$ and $v_{w}=27000 \mathrm{~mm} /$ min.

Fig.12 The influence of speed ratios $v_{w} / n_{s}$ on the morphology of dimple surface at $a_{p}=20 \mu \mathrm{m}$. 


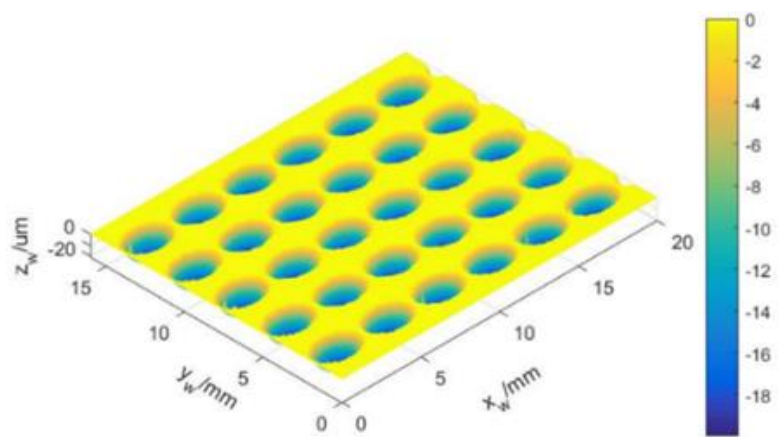

(a) Array pattern

Fig. 11 Simulation diagrams of surface dimple surface with different arrangements at $a_{p}=20 \mu \mathrm{m}$, $n_{s}=1800 \mathrm{r} / \mathrm{min}, v_{w}=23400 \mathrm{~mm} / \mathrm{min}$ and $v_{w} / n_{s}=13$.

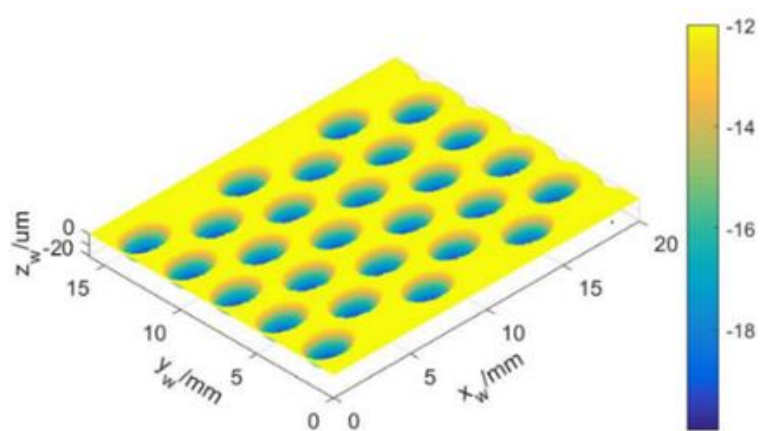

(b) Staggered pattern

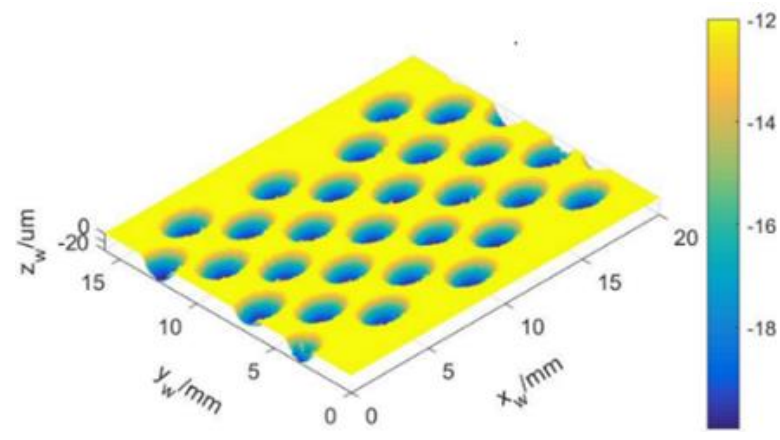

(c) Phyllotactic pattern

Fig. 13 is the dimple surface morphology obtained based on the simulation conditions of Fig. 11a when the grinding depth is reduced to $10 \mu \mathrm{m}$ and $15 \mu \mathrm{m}$, respectively. When the grinding depth is $10 \mu \mathrm{m}, 15 \mu \mathrm{m}$ and $20 \mu \mathrm{m}$, the maximum feature length of the dimple unit is $2.25 \mathrm{~mm}, 2.77 \mathrm{~mm}$ and $2.9 \mathrm{~mm}$, and the width is $1.3 \mathrm{~mm}, 1.7 \mathrm{~mm}$ and $2 \mathrm{~mm}$, respectively. The arrangement cycle in the $X_{w}$ direction is $3.22 \mathrm{~mm}$, and the cycle in the $Y_{w}$ direction remains unchanged at $3 \mathrm{~mm}$.

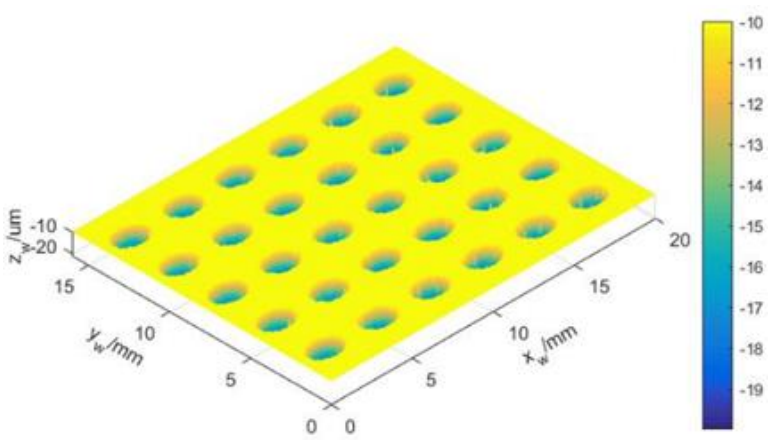

(a) at $a_{p}=10 \mu \mathrm{m}$.

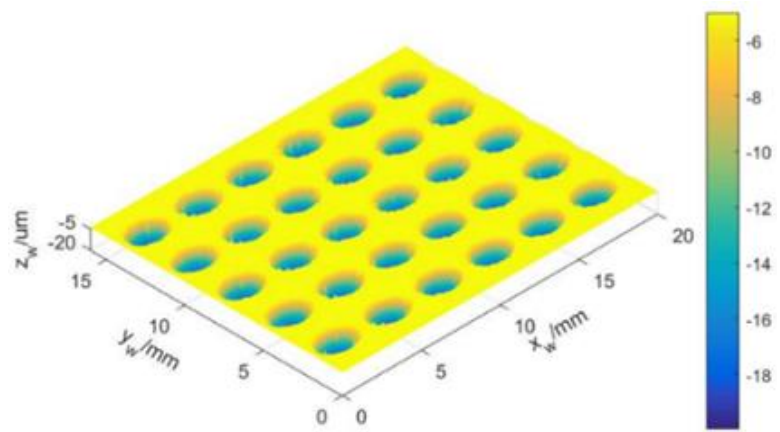

(b) at $a_{p}=15 \mu \mathrm{m}$.

Fig.13 The influence of grinding depth $a_{p}$ on the morphology of dimple surface at $v_{w} / n_{s}=13$, $n_{s}=1800 \mathrm{r} / \mathrm{min}$ and $v_{w}=23400 \mathrm{~mm} / \mathrm{min}$.

The above Figs. 11, 12 and 13 are just a few examples to illustrate the problem. In fact, we have simulated different grinding parameters in the process of designing the grinding wheel. The results show that with the increase of the grinding depth, the dimple arrangement cycle does not change, the length and width of the dimple unit increase, and the growth rate is gradually reduced by the influence of the dimple shape; When the parameters of the grinding wheel are constant, the speed ratio $v_{w} / n_{\mathrm{s}}$ not only affects the length and arrangement period of the dimple units in the $X_{w}$ direction, but also controls the separation, touch 
and intersection between the dimple units, thus changing the morphology of structured dimple surface.

\section{Grinding experiment}

\subsection{Experimental setup}

The grinding experiment was done on the CNC machining center as shown in Fig. 14. The grinding wheel used is shown in Fig. 9, and the structural parameters of the grinding wheel are shown in Tab. 1. The size of the workpiece is $50 \mathrm{~mm} \times 25 \mathrm{~mm} \times 15 \mathrm{~mm}$, the workpiece material is 45 steel, its hardness is HRC36-42, and the initial surface roughness is Ra0.3. The workpiece is fixed on the worktable by a precision vise, and grinding method is up-grinding. No coolant is used during grinding, and the Taylor Hobson Form Talysurf $i$ profiler is used to measure the three-dimensional topography of the ground surface of the workpiece.

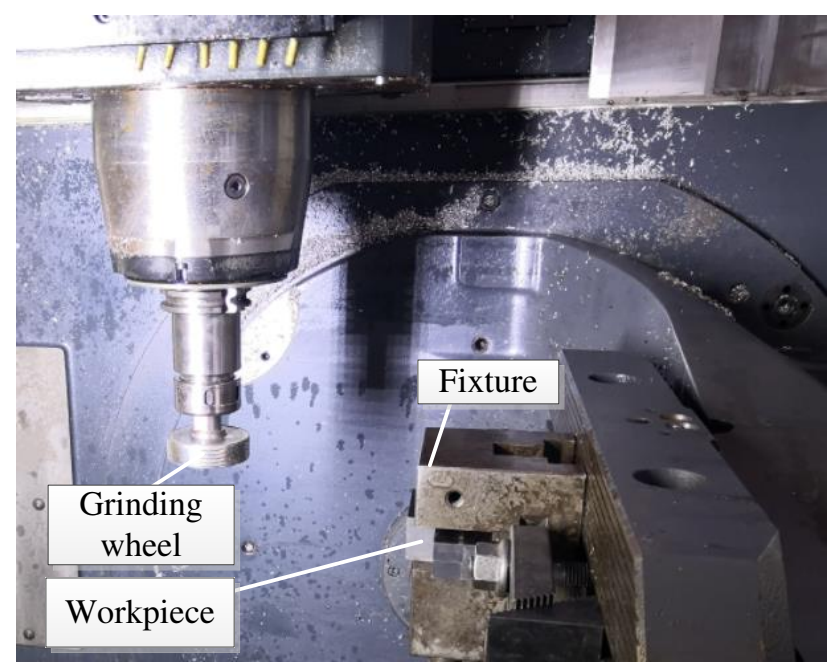

Fig. 14 Experimental setup

\subsection{Experimental results and analysis}

Fig.15 shows the workpiece surface e ground by the grinding wheel with array pattern. The average length of the dimple unit is $3.1 \mathrm{~mm}$, the average width is $1.6 \mathrm{~mm}$, and the average maximum depth is about $12 \mu \mathrm{m}$. The average arrangement cycle in feed speed direction is $3.2 \mathrm{~mm}$, and the cycle in vertical feed speed direction is $3 \mathrm{~mm}$. It can be seen that the arrangement parameters of the ground surface are basically the same as the design parameters, but the feature size of the structured unit has a big difference. Through the microscopic observation and measurement of the ground dimples and the analysis of the grinding process, it is found that the reason for this phenomenon is caused by the abrasive distribution features on the surface of the electroplated CBN grinding wheel. When designing and manufacturing the geometric sizes of the abrasive clusters, the average geometric size of the abrasive grains was taken as a reference, and the protruding height of abrasive grains is assumed to be equal, while the actual protruding height of abrasive grains on the surface of the electroplated grinding wheel obeys a the normal distribution [52,53]. When the theoretical grinding depth $a_{p}=20 \mu \mathrm{m}$ is set based on the spark generated by the grinding wheel, only part of the abrasive particles actually participates in the grinding, so that the surface of the dimple is enveloped by rough grooves, and the actual grinding depth does not reach theoretical grinding depth. Therefore, it also shows that improving the protruding height of abrasive grains of the electroplated grinding wheel is very important to realize this grinding method. 


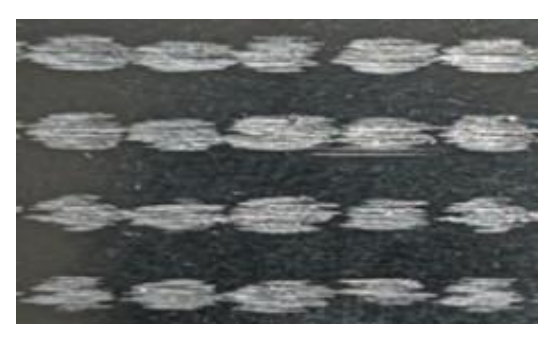

(a) Surface image
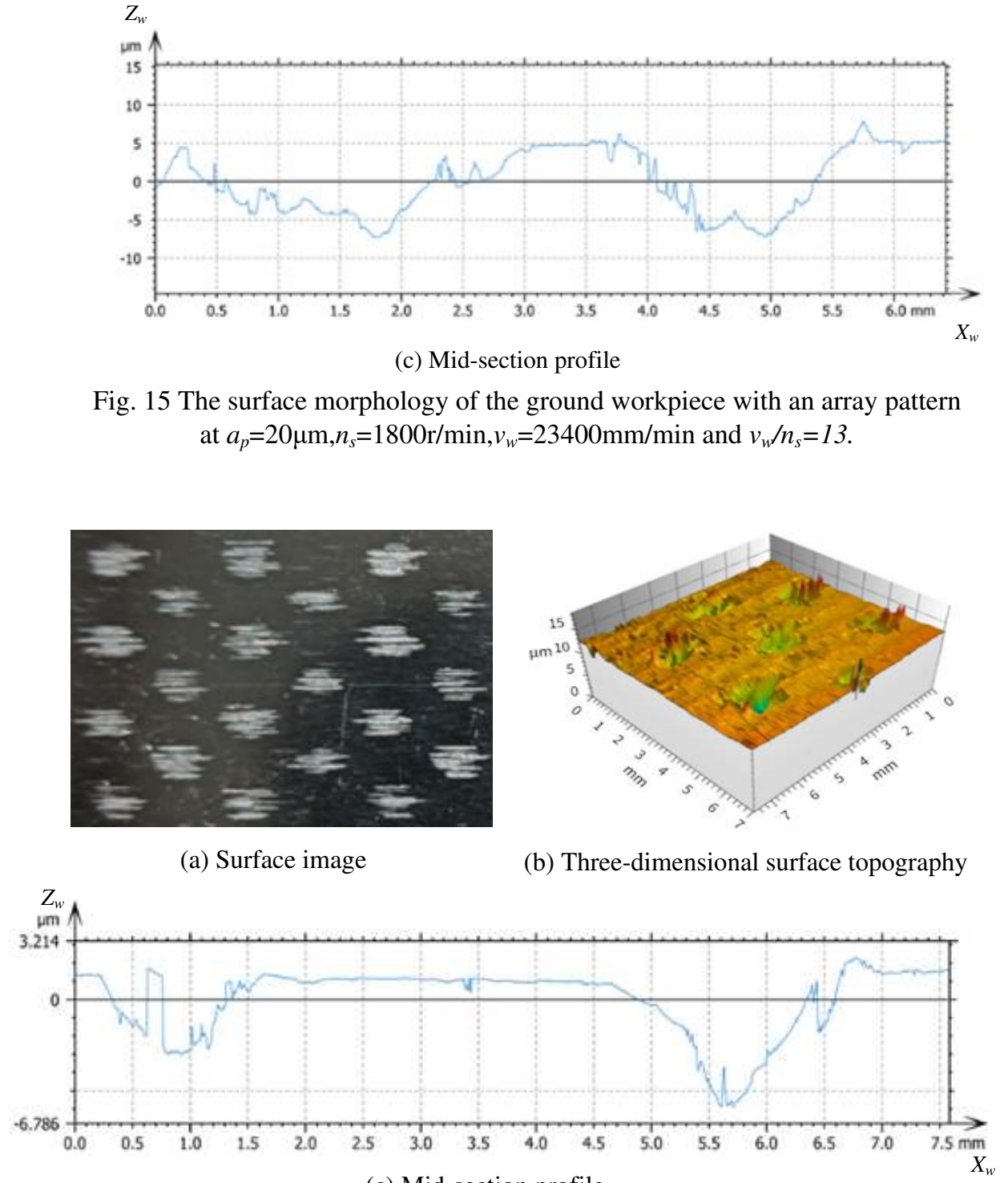

(c) Mid-section profile

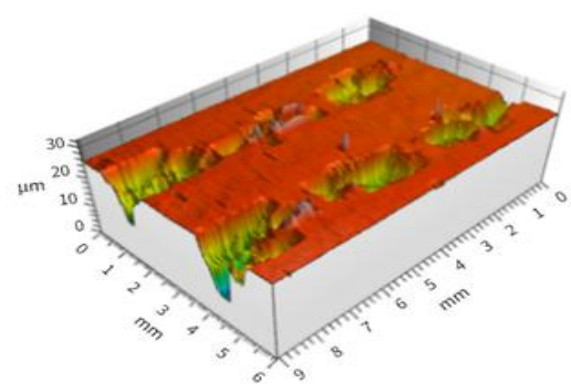

(b) Three-dimensional surface topography (a) Surface image

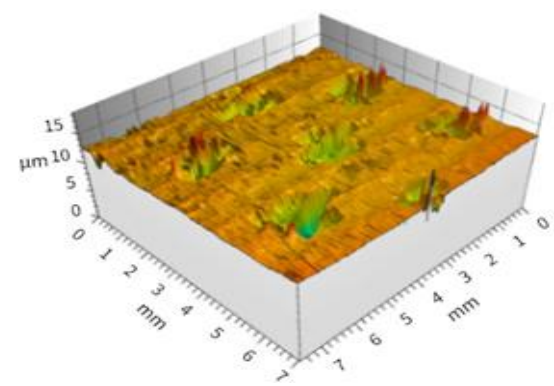

(b) Three-dimensional surface topography

Fig. 16 The surface morphology of the ground workpiece with an staggered pattern at $a_{p}=10 \mu \mathrm{m}, n_{s}=1800 \mathrm{r} / \mathrm{min}, v_{w}=18000 \mathrm{~mm} / \mathrm{min}$ and $v_{w} / n_{s}=10$.

Fig.16 shows the ground dimple surface with staggered pattern when the speed ratio is reduced to $v_{w} / n_{s}=10$ and the grinding depth is reduced to $a_{p}=10 \mu \mathrm{m}$ based on the design parameters. The average length of the dimple unit is $2.2 \mathrm{~mm}$, the average width is $1.6 \mathrm{~mm}$, and the average depth is $8 \mu \mathrm{m}$. The average cycle in the feed speed direction is $5 \mathrm{~mm}$, the average cycle in the direction perpendicular to the feed speed is $3 \mathrm{~mm}$, the phase difference in the feed speed direction is $2.5 \mathrm{~mm}$, and the phase difference in the direction perpendicular to the feed speed is $1.5 \mathrm{~mm}$. By measuring and observing the dimple structure 
of the workpiece surface, it can be known that the topological feature attributes of the dimple surface have not changed, but the topological feature parameters will decrease accordingly. As the grinding depth decreases, the number of abrasive grains involved in the grinding decreases, and the dimpled surface is composed of only a few grinding groove marks of abrasive grains.

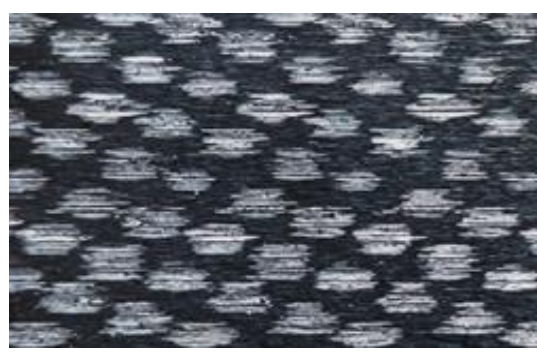

(a) Surface image

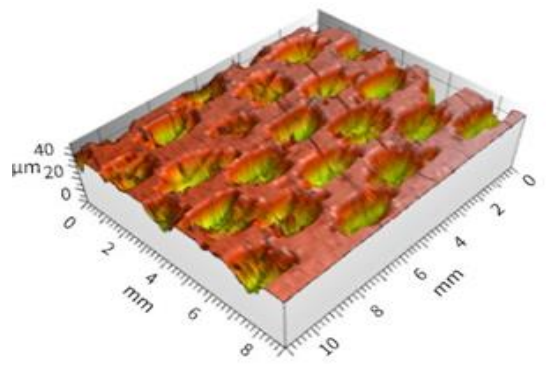

(b) Three-dimensional surface topography

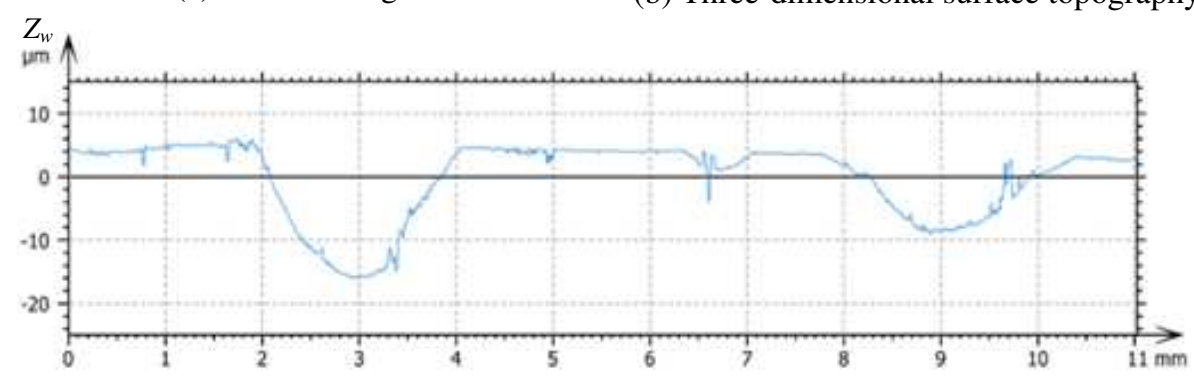

(c) Mid-section profile

Fig. 17 The surface morphology of the ground workpiece with an phyllotactic pattern at $a_{p}=20 \mu \mathrm{m}, n_{s}=1800 \mathrm{r} / \mathrm{min}, v_{w}=18000 \mathrm{~mm} / \mathrm{min}$ and $v_{w} / n_{s}=10$.

Fig. 17 shows the ground dimple surface with the phyllotactic pattern when the speed ratio is reduced to $v_{w} / n_{s}=10$ and the grinding depth is reduced to $a_{p}=20 \mu \mathrm{m}$. The measurement results show that the average length of the dimple unit is $2.7 \mathrm{~mm}$, the average width is $1.6 \mathrm{~mm}$, the average maximum depth of the dimple is $15 \mu \mathrm{m}$, the initial phase difference in the direction of the feed speed is $3.8 \mathrm{~mm}$, and the initial phase difference in the direction of the perpendicular to the feed speed is $0.7 \mathrm{~mm}$. It can also be seen that the topological properties of the dimple surface remain unchanged. However, it can be seen from mapping equation (25) that intersection of dimples should occur on the ground surface by the grinding wheel designed according to Tab. 1 when the speed ratio decreases from $v_{w} / n_{s}=13$ to $v_{w} / n_{s}=10$, but it does not occur in the actual results. The reason is still the normal distribution of the actual protruding height of abrasive grains on the surface of grinding wheel, and the aligning reference of the grinding depth is based on the highest abrasive point, which results in the actual grinding depth being less than $20 \mu \mathrm{m}$ of the theoretical grinding depth. 


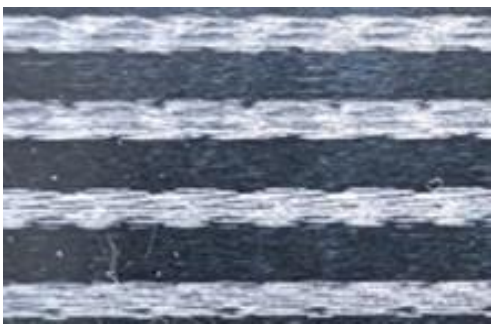

(a) Surface image

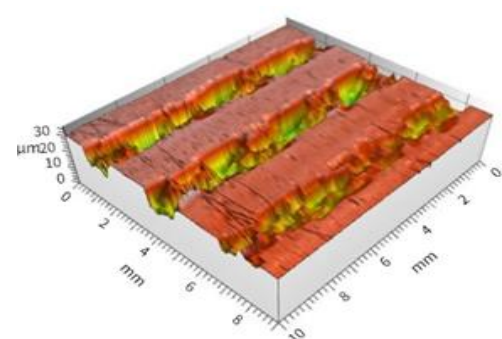

(b) Three-dimensional surface topography

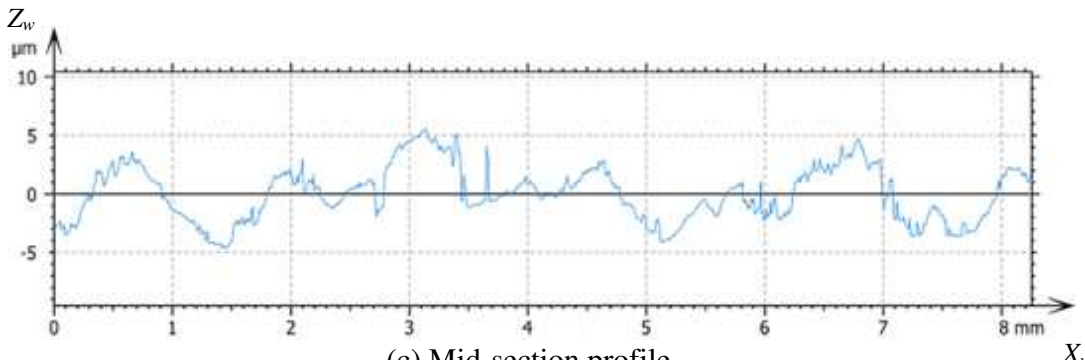

(c) Mid-section profile

Fig. 18 The surface morphology of the ground workpiece with an array pattern at $a_{p}=20 \mu \mathrm{m}, n_{s}=1800 \mathrm{r} / \mathrm{min}, v_{w}=10800 \mathrm{~mm} / \mathrm{min}$ and $v_{w} / n_{s}=6$.

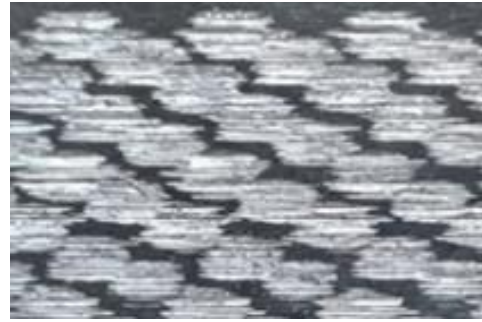

(a) Surface image

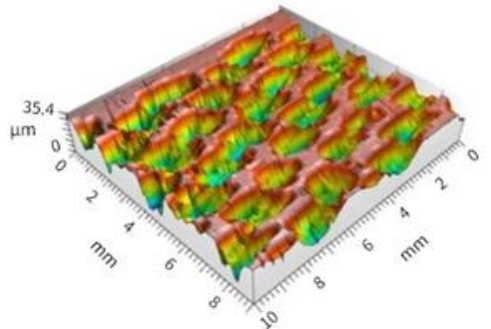

(b) Three-dimensional surface topography

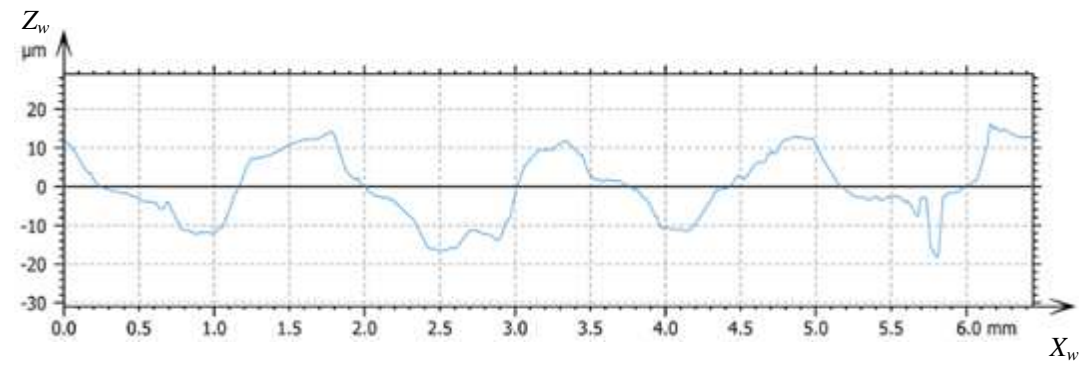

(c) Mid-section profile

Fig. 19 The surface morphology of the ground workpiece with an phyllotactic pattern at $a_{p}=20 \mu \mathrm{m}, n_{s}=1800 \mathrm{r} / \mathrm{min}, v_{w}=10800 \mathrm{~mm} / \mathrm{min}$ and $v_{w} / n_{s}=6$.

Fig. 18 and Fig. 19 respectively show the surface morphology of the workpiece ground by the grinding wheel with array pattern and phyllotactic pattern when the speed ratio is reduced from $v_{w} / n_{s}=13$ to $v_{w} / n_{s}=6$. At this time, the dimple surface ground by the grinding wheels with array pattern has already produced an intersecting shape along the feed speed direction, and the corresponding arrangement properties have basically disappeared; the dimple surface ground by the grinding wheel with phyllotactic pattern has produced intersections between the dimples in the vertical direction of the feed speed, but certain topological properties of the arrangement can still be observed. These also indicate that the selection of grinding parameters at this time cannot satisfy the kinematic conditions of generating the dimple surface expressed by Eqs. (20) and (21). However, it can be seen that due to the reduction of the 
feed speed of the workpiece, the dynamic grinding edge participating in the grinding of the dimples increases [48,53], and the cutting groove marks on the surface of the dimples become dense. The actual depth of the dimple increases and the bottom surface becomes smoother, as shown in Fig. 19.

\section{Conclusions and Outlook}

Based on the geometric topology theory, the mapping relationship matrix between the topological space of workpiece and the topological space of the grinding wheel is established. The structured grinding wheels with abrasive clusters are designed and the analysis and geometric simulation of the surface creation in the topological grinding process are performed. At the same time, the grinding wheel was manufactured and the grinding experiment was carried out. The results show that the topological grinding of the structured dimple surface can be realized by the designed grinding wheel with ordered abrasive clusters based on the topological properties of the dimple surface. Although the ground dimple structured surface is an approximation of the theoretical surface, it still maintains the topological properties unchanged. To achieve the grinding of the structured dimple surface, the selection of grinding parameters must meet the corresponding kinematics conditions. These studies provide a new way for the grinding of the structured surface.

In the follow-up work, the manufacturing quality of grinding wheel should be further improved, such as improving the manufacturing accuracy of the substrate and improving the contour of the protruding height of abrasive grains on the surface of the grinding wheel; the feasibility study of topological grinding for different structured surfaces will also be carried out. The grinding wheel is designed more closely based on the functional feature parameters of the structured surface, and this grinding method is realized, and the precision relationship between the ground topological surface and the designed workpiece surface will be explored in depth.

\section{Statements \& Declarations}

\section{Funding}

This work was supported by National Natural Science Foundation of China (Grant numbers: 51875368)

\section{Competing Interests}

Financial interests: Author Yushan Lyu and Guoxun Wang and Xingshan Li and Yongchao Qi declare they have no financial interests.

\section{Author Contributions}

All authors contributed to the study conception and design. Material preparation, data collection and analysis were performed by Guoxun Wang, Xingshan Li and Yongchao Qi. The first draft of the manuscript was written by Yushan Lyu and all authors commented on previous versions of the manuscript. All authors read and approved the 
final manuscript.

\section{Availability of data and material}

The datasets used and/or analyzed during the current study are available from the corresponding author on reasonable request.

\section{Code availability}

Not applicable

\section{Ethics approval}

Written informed consent for publication of this paper was obtained from the Shenyang Ligong University and all authors.

\section{Consent to participate}

Not applicable

\section{Consent for publication}

The Author confirms: that the work described has not been published before ; that it is not under consideration for publication elsewhere; that its publication has been approved by all co-authors; that its publication has been approved by the responsible authorities at the institution where the work is carried out.

The Author agrees to publication in The International Journal of Advanced Manufacturing Technology and also to publication of the article in English by Springer in Springer's corresponding English-lanquage journal. The copyright to the English-lanquage article is transferred to Springer effective if and when the article is accepted for publication.

\section{References}

1. Evans C J, Bryan J B (1999) "Structured", "textured" or "engineered" surfaces. CIRP Annals Manufacturing Technology, 48(2):541-556.

2. Malshe A P, Bapat S, Rajurkar K P, Haitjema H (2018) Bio-inspired textures for functional applications. CIRP Annals - Manufacturing Technology 67(2):627-650.

3. Han Z W, Mu Z Z, Yin W (2016) Biomimetic multifunctional surfaces inspired from animals, Advances in Colloid and Interface Science 234: 27-50.

4. Liu K s, Lei J (2011) Bio-inspired design of multiscale structures for function integration, Nano Today 6:155-175

5. Yu C M, Liu M F, Zhang C H, et al (2020) Bio-inspired drag reduction: From nature organisms to 
artificial functional. Giant 2 100017:1-12.

6. Luo Y H, Yuan L, Li J H, Wang J S (2015) Boundary layer drag reduction research hypotheses derived from bio-inspired surface recent advanced applications. Micron. 79: 59-73.

7. Ibatan T, Uddin M S, Chodhury M A K (2015) Recent development on surface texturing in enhancing tribological performance of bearing sliders. Surface \& Coatings Technology 272:102-120.

8. Liu X M, Zhao D C, Chen L, et al (2019) Biomimetic biological tribology development status and application prospect. Chinese Journal of Construction Machinery 017(002):95-101.

9. Brinksmeier E, Karpuschewski B, Yan J, Schönemann L (2020) Manufacturing of multiscale structure surface. CIRP Annals - Manufacturing Technology 69(2):717-739.

10. Hirt G, Thome M (2008) Rolling of functional metallic surface structures, CIRP Annals Manufacturing Technology 57:317-320.

11. Schneider Y G (1984) Formation of surfaces with uniform micro-patterns on precision machine and instruments parts. Precis Eng 6(4):219-25.

12. Bai L J, Zhang J H, Tao G C, et al ( 2016 ) Vibration Assisted milling for bionic surface manufacturing. China Mechanical Engineering 27(009):1229-1233,1242.

13. Wang X Z, Duan J, Jiang M, Ke S H, et al (2017) Study of laser precision ablating texture patterns on large-scale freeform surface. Int J Adv Manuf Technol 92:4571-4581.

14. Saito Y, Okamoto S, Miki A, et al (2008) Fabrication of micro-structure on glass surface using micro-indentation and wet etching process. Applied Surface Science 254(22):7243-7249.

15. Stepien Piotr (1989) Generation of regular patterns on ground surfaces. CIRP Annals Manufacturing Technology. 38 (1):561-566.

16. Stepien Piotr (2007) Grinding forces in regular surface texture generation. International Journal of Machine Tools \& Manufacture 47:2098-2110.

17. Stepien Piotr (2008) Mechanism of grinding wheel surface reproduction in regular surface texture generation. Surface Engineering 24(3): 219-225.

18. Stepien Piotr (2009) Regular surface texture generated by special grinding process. J Manuf Sci Eng 131(1):011015-1011015-7.

19. Stepien Piotr (2011) Deterministic and stochastic components of regular surface texture generated by a special grinding process. Wear 271:514-518.

20. Islam M M, Ko T J (2016) Formulating CAM parameters for surface patterning by grinding process based on unit pattern geometry. Int J Adv Manuf Technol 83:595-609.

21. Kim H C, Ko T J (2014) Simulation of micro-patterns engraved by grinding process with screw shaped wheel. Simulation Modelling Practice and Theory 49:277-286.

22. Kim H C, Ko T J (2015) Verification of simulation of surface texturing on planar surface by grinding. International Journal of Precision Engineering and Manufacturing 16(2):225-231.

23. Cao H Y, Chen X, Li H L (2018) Dressing strategy and grinding control for cylindrical microstructural surface. Int J Adv Manuf Technol 99:707-727.

24. Liu Y M, Gong S, Li J Y, Cao J G (2017) Effects of dressed wheel topography on patterned surface textures and grinding force. Int J Adv Manuf Technol 93:1751-1760.

25. Mohamed AL-M, Warkentin A, Bauer R (2017) A Prediction of workpiece surface texture using circumferentially grooved grinding wheels. Int J Adv Manuf Technol. 89:1149-1160.

26. Oliveira J F G, Bottene A C, Franc T V (2010) A novel dressing technique for texturing of ground surfaces, CIRP Annals - Manufacturing Technology 59 361-364.

27. Silva E J, Oliveira J F G, Salles B B (2013) Strategies for production of parts textured by grinding 
using patterned wheels. CIRP Annals - Manufacturing Technology 62 (1):355-358.

28. Silva E J, Bottene A C, Oliveira J F G (2016) Grinding process for profiled texturing. CIRP Annals Manufacturing Technology 65 (1): 337-340.

29. Silva E J, Kirsch B, Bottene A C, Simon A, Aurich J C, Oliveira J F G (2016) Manufacturing of structured surfaces via grinding. Journal of Materials Processing Technology 243: 170-183.

30. Moreno M G, Ruiz J Á, Azpeitia D B et al (2020) Friction improvement via grinding wheel texturing by dressing. Int J Adv Manuf Technol 107:4939-4954.

31. Denkena B, Köhler J, Wang B (2010) Manufacturing of functional riblet structures by profile grinding. CIRP Journal of Manufacturing Science and Technology 3 (1):14-26.

32. Denkena B, Köhler J, Wang B (2010) Advanced microstructures and its production through cutting and grinding. CIRP Annals - Manufacturing Technology 59 (1):67-72.

33. Denkena B, de Leon L, Wang B (2009) Grinding of microstructured functional surfaces: a novel strategy for dressing of microprofiles. Production Engineering Research and Development 3(1):41-48.

34. Denkena B, Grove T, Krawczyk T (2016) Tools and strategies for grinding of riblets on freeformed compressor blades. Procedia CIRP 55:182-187.

35. Xie J, Luo M J, He J L, et al (2012) Micro-grinding of micro-groove array on tool rake surface for dry cutting of titanium alloy. International Journal of Precision Engineering and Manufacturing 13:1845-1862.

36. Xie J, Li P, Wu K K (2013) Micro and precision grinding technique and functional behavior development of micro-structured surface. Journal of Mechanical Engineering 49 (23):182-190.

37. Wu M T, Guo B, Zhao Q L, Zhang J, Fang X Y, He P (2019) High efficiency precision grinding of micro-structured $\mathrm{SiC}$ surface using laser micro-structured coarse-grain diamond grinding wheel. International Journal of Precision Engineering and Manufacturing-Green Technology 6:577-586.

38. Li H N, Axinte D (2016) Textured grinding wheels: A review. International Journal of Machine Tools \& Manufacture 109:8-35

39. Monier A, Guo B, Zhao Q L, Liu W C (2021) Strategy and error analysis for machining the designed micro structured surfaces by structured grinding wheels. Int J Adv Manuf Technol 113:1361-1376.

40. Xu S L, Point Set Topology, Higher Education Press, July 1, 2007.

41. Galda L, Dzierwa A, Sep J, Pawlus P (2010) The effect of oil pockets shape and arrangement on seizure resistance in lubricated sliding. Tribol. Lett. 37: 301-311.

42. Nakano M, Korenaga A, Korenaga A, Miyake K et al (2007) Applying micro-texture to cast iron surfaces to reduce the friction coefficient under lubricated conditions. Tribol. Lett. 28 131-137.

43. Li K, Yao Z, Hu Y, Gu W (2014) Friction and wear performance of laser peen textured surface under starved lubrication .Tribol. Int. 77 97-105.

44. Lu Y S, Liu Y M, Wang J, Liu H P (2016) Tribological performance of dimpled thrust bearings with phyllotactic patterns. Wear 346-347:108-115.

45. Lu Y S, Liu Y M, Wang J, Liu H P (2014) Experimental investigation into friction performance of dimples journal bearing with phyllotactic pattern. Tribol. Lett. 55 (2): 271-278.

46. Iterson G Van, Mathematische und mikroskopisc-anatomische Studien u"ber Blattstellungen nebst

Betrachtungen u"ber den Schalenbau der Miliolin.1907.

47. Lyu Y S, Liu Y M, Li X S, Di H X, Wang H, (2021) A strategy on generating structured plateau 
surface by the sinusoidal oscillatory lapping of the grinding wheel with the phyllotactic pattern of abrasive grains. Journal of Manufacturing Processes 65 435-444

48. Ren J S, Principles of grinding, Publishing House of Electronics Industry, January 1, 2011.

49. Li X S, Lü Y S, Wang Y C (2021) The Strategy of grinding dimpled surface for reducing friction force by grinding wheel with ordered abrasive grain clusters. Acta Armamenta R II 42(3):633-639.

50. Liu Z Z, Wang J, Lu Y S, Ma F (2014) Fabrication of the electroplated CBN wheel for cylindrical grinding with abrasive phyllotactic pattern. Advanced Materials Research. 1077:44-49.

51. Ma F, Lu Y S, Wang J (2014) The Fabrication of the convex platform surface with phyllotactic pattern by electrolytic etching method. Applied Mechanics and Materials 685:85-89.

52. Shi Z, Malkin S (2006) Wear of Electroplated CBN Grinding Wheels. Transactions of the ASME, Journal of Manufacturing Science and Engineering 128: 110-118.

53. Yu H Y, Wang J, Lu Y S (2016) Modeling and analysis of dynamic cutting points density of the grinding wheel with an abrasive phyllotactic pattern. Int J Adv Manuf Technol 86:1933-1943. 